\title{
Molecular Basis of Action of a Small-Molecule Positive Allosteric Modulator Agonist at the Type 1 Cholecystokinin Holoreceptor $\$$
}

\author{
Aditya J. Desai, Ingrid Mechin, Karthigeyan Nagarajan, Celine Valant, Denise Wootten, \\ Polo C. H. Lam, Andrew Orry, Ruben Abagyan, Anil Nair, Patrick M. Sexton, \\ Arthur Christopoulos, and Laurence J. Miller \\ Department of Molecular Pharmacology and Experimental Therapeutics, Mayo Clinic, Scottsdale, Arizona (A.J.D., L.J.M.); In \\ Silico Drug Discovery Department, Icagen Tucson Innovation Center, Oro Valley, Arizona (I.M., K.N., A.N.); Drug Discovery \\ Biology Theme, Monash Institute of Pharmaceutical Sciences and Department of Pharmacology, Monash University, Parkville, \\ Australia (C.V., D.W., P.M.S., A.C., L.J.M.); Molsoft, La Jolla, California (P.C.H.L., A.O., R.A.); Skaggs School of Pharmacy and \\ Pharmaceutical Sciences, University of California, San Diego, La Jolla, California (R.A.); and School of Pharmacy, Fudan \\ University, Shanghai, China (D.W., P.M.S.)
}

Received August 25, 2018; accepted December 19, 2018

\begin{abstract}
Allosteric modulation of receptors provides mechanistic safety while effectively achieving biologic endpoints otherwise difficult or impossible to obtain by other means. The theoretical case has been made for the development of a positive allosteric modulator (PAM) of the type 1 cholecystokinin receptor (CCK1R) having minimal intrinsic agonist activity to enhance meal-induced satiety for the treatment of obesity, while reducing the risk of side effects and/or toxicity. Unfortunately, such a drug does not currently exist. In this work, we have identified a PAM agonist of the CCK1R, SR146131, and determined its putative binding mode and receptor activation mechanism by combining molecular modeling, chimeric CCK1R/CCK2R constructs, and sitedirected mutagenesis. We probed the structure-activity
\end{abstract}

relationship of analogs of SR146131 for impact on agonism versus cooperativity of the analogs. This identified structural features that might be responsible for binding affinity and potency while retaining PAM activity. SR146131 and several of its analogs were docked into the receptor structure, which had the natural endogenous peptide agonist, cholecystokinin, already in the bound state (by docking), providing a refined structural model of the intact CCK1R holoreceptor. Both SR146131 and its analogs exhibited unique probedependent cooperativity with orthosteric peptide agonists and were simultaneously accommodated in this model, consistent with the derived structure-activity relationships. This provides improved understanding of the molecular basis for CCK1R-directed drug development.

\section{Introduction}

Cholecystokinin (CCK) has multiple important physiologic functions, including stimulating gallbladder contraction and pancreatic exocrine secretion, regulating gastrointestinal transit, and inducing satiety in response to ingestion of a meal. Indeed, the satiety effect of CCK, mediated by the type 1 CCK receptor (CCK1R), has been demonstrated in both animal models (Gibbs et al., 1973; Chaudhri et al., 2008) and humans (Kissileff et al., 1981), supporting this receptor as a possible target for the treatment of obesity. Substantial efforts

This work was partially supported by the Mayo Clinic and by a grant from the Flinn Foundation. D.W., P.M.S., and A.C. are Career Development, Principal, and Senior Principal Research Fellows, respectively, of the National Health and Medical Research Council of Australia.

https://doi.org/10.1124/mol.118.114082.

S This article has supplemental material available at molpharm. aspetjournals.org. to develop small-molecule agonists of the CCK1R have been undertaken by major pharmaceutical companies (Aquino et al., 1996; Berger et al., 2008; Elliott et al., 2010); however, none of the agents have advanced beyond Phase II clinical trials. These efforts have been hampered by on-target side effects of potent and longer duration agonists, with lower potency agents having insufficient efficacy. A robust therapeutic window is particularly important because of the anticipated scale and duration of use.

A possible strategy to circumvent the safety and tolerability concerns is the development of a pure positive allosteric modulator (PAM) of the CCK1R that is devoid of intrinsic agonist activity (Desai et al., 2015a,b, 2016a,b; Miller and Desai, 2016). Such an agent would bind to the receptor at an allosteric site that is distinct from the natural orthosteric ligand-binding site, allowing concurrent occupation and potentiation of biologic responses to CCK. This would occur only

ABBREVIATIONS: $\Delta \mathrm{G}$, relative binding affinities; BDZ-1, (S)-1-(3-iodophenyl)-3-(1-methyl-2-oxo-5-phenyl-2,3-dihydro-1H-benzo[e][1,4]diazepin3-yl)urea; BDZ-2, (R)-1-(3-iodophenyl)-3-(1-methyl-2-oxo-5-phenyl-2,3-dihydro-1H-benzo[e][1,4]diazepin-3-yl)urea; CCK, cholecystokinin; CCK1R or CCK2R, type 1 or 2 CCK receptor; CHO, Chinese hamster ovary; ECL, extracellular domain; GPCR, G protein-coupled receptor; GLP-1, glucagon-like peptide-1; ICM, internal coordinate mechanics; KRH, Krebs-Ringer-HEPES; mmGBSA, molecular mechanics, the generalized Born model and solvent accessibility method; OPE, O-phenylethyl ester; PAM, positive allosteric modulator; TM, transmembrane domain; WT, wild-type. 
during the limited and finite period of time when nutrients enter the duodenum to stimulate the release of endogenous hormone, resulting in amplification of the natural effects of CCK. Such a mechanism of action would be predicted to lead to an early peak in satiety, reducing meal size and contributing to body weight reduction, while, in the meantime, minimizing the side effects observed with full agonists of the CCK1R and avoiding the tolerance often experienced with such agonists.

A small-molecule ligand-binding site within the CCK1R has been localized to a pocket high within the helical transmembrane domain using receptor mutagenesis, fluorescence, and photoaffinity-labeling approaches (Hadac et al., 2006; Cawston et al., 2012; Harikumar et al., 2013; Desai et al., 2015c). This pocket assumes distinct conformations conferring different molecular determinants of ligand binding in its inactive state occupied by antagonist ligands (Cawston et al., 2012), versus its active state occupied by agonist ligands (Harikumar et al., 2013; Desai et al., 2015c).

We previously proposed an experimental strategy in which a key chemical group (isopropyl), identified in small-molecule structure-activity studies as critical for its agonist activity, would be changed or eliminated to reduce its intrinsic agonist activity, while theoretically stabilizing a conformation that reduces the energy barrier of activation, and possibly exhibiting PAM activity (Desai et al., 2015b). However, the first application of this strategy to GI181771X, an allosteric agonist lacking intrinsic PAM activity, was unsuccessful in achieving PAM activity, and instead resulted in a negative allosteric modulator of CCK action (Desai et al., 2015b). We postulated that these analogs could adopt poses in the allosteric pocket that would be distinct from that of the parent compound (Desai et al., 2015b).

In the current work, we identified the small-molecule ligand, SR146131 (Bignon et al., 1999), as a selective CCK1R PAM agonist that could form a promising template for differentiation of structure-activity driving intrinsic efficacy versus allosteric enhancement. SR146131 differed from an antagonist in the same series, SR27897, possessing a cyclohexylethyl group attached to its thiazol ring, as well as two methyl groups on the indole ring and two methoxy groups on the phenyl ring (Gouldson et al., 2000). Moreover, SR146131 enhanced the potency of functional responses to the orthosteric ligand, CCK. Docking of the compound into a previously developed molecular model of CCK1R in its active state (Harikumar et al., 2013) predicted key interactions between the 5-methoxy group on the phenyl ring of SR146131 with transmembrane segment residue Leu 7.39 of CCK1R that we had previously linked to the agonist activity of GI181771X. Consequently, we studied a series of structural analogs to provide insights into the molecular basis of agonism and cooperativity.

Included in this series of compounds was an analog with attenuated agonist activity, reflected in lower binding affinity, which retained its positive cooperativity on CCK action. This ligand was docked into a refined molecular model that accommodated simultaneous binding of natural CCK peptide. Within the compound series, the PAM activity was correlated with its intrinsic efficacy, thus identifying structural features for binding affinity independent of efficacy and allosteric modulation. Although future work will focus on the potential to separate intrinsic efficacy and positive modulation, the current study advances our understanding of the molecular determinants for modulator binding that may be useful in the design and development of ligands with tailored molecular properties.

\section{Materials and Methods}

Materials. Ham's F-12 medium, OptiMEM medium, L-glutamine, Lipofectamine LTX, and PLUS reagents were from Invitrogen (Carlsbad, CA). Quest Fluo-8-AM was from AAT Bioquest (Sunnyvale, CA). Fetal clone II tissue culture medium supplement was from Hyclone Laboratories (Logan, UT). Microscint, Unifilter 96-well microplates with bonded GF/B filters were from PerkinElmer Life and Analytical Sciences (Shallon, CT). Costar 96-well V-bottom assay plates and the black assay plates with clear bottoms were from Corning (Corning, NY). All other reagents were analytical grade.

Ligands. SR146131, 2-[4-(4-chloro-2,5-dimethoxyphenyl)-5-(2cyclohexyl-ethyl)-thiazol-2-ylcarbam-oyl]-5,7-dimethyl-indol-1-yl-1acetic acid, was provided by Sanofi (structure shown in Fig. 1A). This ligand is a potent full agonist at the CCK1R (Bignon et al., 1999; Gouldson et al., 2000). Cholecystokinin octapeptide (CCK-26-33, based on the numbering of CCK-33; also known as CCK-8 and commonly identified as CCK) was purchased from Peninsula Laboratories (Belmont, CA). The O-phenylethyl ester (OPE) analog of CCK, previously demonstrated to be a partial agonist at the CCK1R (CCKOPE) (Gaisano et al., 1989), was synthesized in our laboratory. The CCK1R-selective 1,4-benzodiazepine radioligand $\left[{ }^{125} \mathrm{I}-(\mathrm{S})-1-(3-\right.$ iodophenyl)-3-(1-methyl-2-oxo-5-phenyl-2,3-dihydro-1H-benzo[e][1,4] diazepin-3-yl)urea (BDZ-1)] and the type 2 CCK receptor (CCK2R)selective radioligand $\left[{ }^{125} \mathrm{I}-(\mathrm{R})-1\right.$-(3-iodophenyl)-3-(1-methyl-2-oxo-5phenyl-2,3-dihydro-1H-benzo[e][1,4] diazepin-3-yl)urea (BDZ-2)] were prepared, as we described previously (Akgün et al., 2009). These were radioiodinated using oxidative techniques with iodobeads (Pierce, Rockford, IL), and the products were purified to homogeneity on reversed-phase highpressure liquid chromatography (Powers et al., 1988). Both radioligands had specific radioactivities of approximately $2000 \mathrm{Ci} / \mathrm{mmol}$.

CCK Receptor-Bearing Cell Lines and Membrane Preparation. Chinese hamster ovary (CHO) cell lines stably expressing a series of CCK receptor constructs that have been previously characterized (Cawston et al., 2012; Harikumar et al., 2013; Desai et al., $2015 \mathrm{~b}$ ) were used in the current studies. The cell lines were passaged approximately twice per week and were maintained at $37^{\circ} \mathrm{C}$ in Ham's F-12 medium supplemented with 5\% Fetal Clone II in a humidified environment containing 5\% carbon dioxide. As described previously (Hadac et al., 1996), receptor-bearing membrane fractions were prepared using homogenization and sucrose-density gradient centrifugation, with the resulting particulate fraction suspended in KrebsRinger-HEPES (KRH) medium (in millimolars, 25 HEPES, $\mathrm{pH} 7.4$, $104 \mathrm{NaCl}, 5 \mathrm{KCl}, 1.5 \mathrm{CaCl}_{2}, 1.0 \mathrm{KH}_{2} \mathrm{PO}_{4}, 1.2 \mathrm{MgSO}_{4}, 1.2 \mathrm{MgCl}_{2}$ ) containing $0.01 \%$ soybean trypsin inhibitor and $1 \mathrm{mM}$ phenylmethylsulfonyl fluoride and stored at $-80^{\circ} \mathrm{C}$ until use.

Receptor-Binding Assays. Individual radioligand competitionbinding assays were performed in duplicate using benzodiazepine radioligands (Cawston et al., 2012; Harikumar et al., 2013; Desai et al., 2015b). Briefly, $\sim 5-7 \mu \mathrm{g}$ membranes were added to a $100 \mu \mathrm{l}$ assay volume in a clear 96-well plate containing increasing concentrations of SR146131 and $\sim 20-25 \mathrm{pM}$ radioligand, in KRH medium, and incubated for 60 minutes at room temperature. The reaction was then terminated by vacuum filtration using Unifilter 96-well microplates with bonded GF/B filters in a Filtermate Harvester (PerkinElmer, Waltham, MA). Nonsaturable binding was determined using the corresponding unlabeled ligand, $1 \mu \mathrm{M}$ BDZ-1 or BDZ-2. The plates were washed six times using $0.9 \% \mathrm{NaCl}$ and $0.2 \%$ bovine serum albumin and then dried, with radioactivity quantified using a Top CountNXT instrument (Packard, Meriden, CT) after addition of $30 \mu \mathrm{l}$ Microscint.

Intracellular Calcium Assays. CCK receptor-mediated biologic responses were measured by quantifying intracellular calcium increases in response to SR146131 (Desai et al., 2014, 2015b). Briefly, 


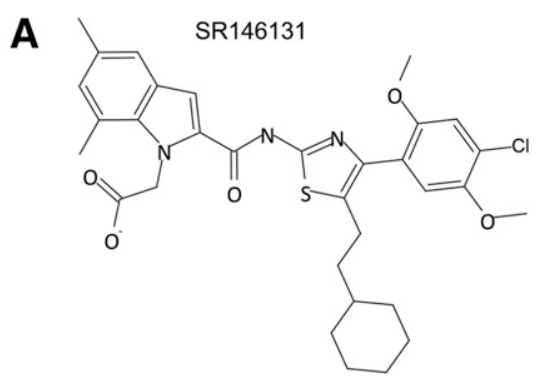

B

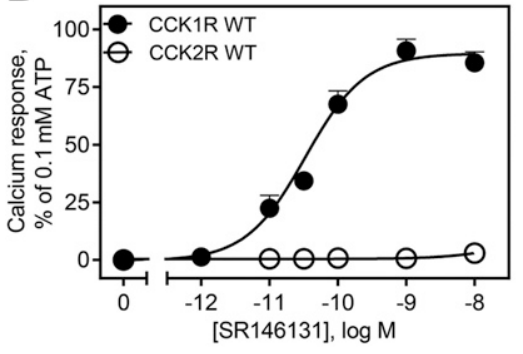

C

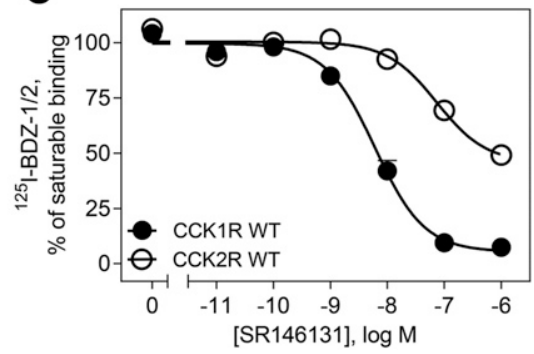

Fig. 1. Properties of SR146131. Shown is the structure of SR146131 (A), along with intracellular calcium responses generated at both subtypes of CCK receptors stably expressed on CHO cells (B). Data are represented as means \pm S.E.M. of eight experiments. Shown also are the data from competition-binding studies with the allosteric antagonist radioligands, ${ }^{125} \mathrm{I}-\mathrm{BDZ}-1$ at the CCK1R or ${ }^{125} \mathrm{I}-\mathrm{BDZ}-2$ at the CCK2R stably expressed on CHO cell membranes (C). Nonsaturable binding was determined by using $1 \mu \mathrm{M}$ unlabeled BDZ. Data are represented as means \pm S.E.M. of data from six experiments for CCK1R and five experiments for CCK2R. cells were seeded in sterile clear-bottom black 96 -well tissue culture plates 24 hours before the assay to achieve $75 \%-80 \%$ confluence at the time of the assays. Cells were loaded with $0.75 \mu \mathrm{M}$ Fluo-8-AM dye in $\mathrm{KRH}$ medium containing $2.5 \mathrm{mM}$ probenecid for 45 minutes at $37^{\circ} \mathrm{C}$ in the dark. They were washed once, and the assay was performed in a Flexstation 3.0 plate reader (Molecular Devices, Sunnyvale, CA) using robotic addition of the appropriate agonist ligand. Intracellular calcium responses were quantified by measuring fluorescence emission intensity at $525 \mathrm{~nm}$ after excitation at $485 \mathrm{~nm}$, with data collection every 4 seconds over a 120 -second period, while maintaining a constant temperature of $37^{\circ} \mathrm{C}$. Concentration-response curves were constructed by plotting the peak responses. To test their positive allosteric effect, fixed concentrations of compounds were added simultaneously with increasing concentrations of CCK, or the partial agonist, CCK-OPE, which is a phenylethyl ester analog of CCK (Gaisano et al., 1989), and intracellular calcium measurements were taken, as mentioned above.

Molecular Modeling. SR146131, together with 159 known CCK1R agonists and 1739 other CCK1R ligands (not identified as agonists) collected from ChEMBL_15, were docked to the previously generated CCK1R agonist receptor models (Harikumar et al., 2013; Desai et al., 2015c) using MolSoft internal coordinate mechanics (ICM) 3.8 (Totrov and Abagyan, 1997). The best agonist receptor model achieved an area under the receiver operating characteristic curve value of $87 \%$, effectively differentiating the agonists from other ligands. We clustered the docking poses of all agonists using the ICM atomic property field method (Totrov, 2008) with a distance cutoff of 0.4. The majority of the CCK1R agonists docked into two distinct docking pose families, consistent with their chemotype (benzodiazepine core: 67 members; piperazine core: 64 members), indicating the general applicability and consistency of the receptor model for docking. SR146131 achieved an ICM docking score of -39, more favorable than the ICM docking score of -32 for a typical binder.

We also developed a refined CCK1R homology model using the recently solved structure of the orexin-1 receptor in complex with the selective antagonist SB-674042 (Protein Data Bank code: 4ZJ8) (Yin et al., 2016) as a template, because this receptor is closer in sequence to CCK1R than the A2a adenosine receptor used in our previous CCK1R model. The orexin-1 receptor has $33 \%$ sequence identity $/ 53 \%$ homology with CCK1R, versus $26 \%$ sequence identity $/ 42 \%$ homology for the A2a adenosine receptor. Although 4ZJ8 is in an antagonist-bound conformation, it also offers the advantage of presenting a preformed ligand-binding site. The starting homology model was generated using Prime (Jacobson et al., 2004) after multiple sequence alignment of several human and mouse class A G protein-coupled receptor (GPCR) sequences. The NMR structure of the amino terminus of CCK1R (Protein Data Bank code: 1D6G) (Pellegrini and Mierke, 1999) was then grafted to the core helical bundle domain. Extracellular loops for the new model were sampled using Loop Modeler from Molecular Operating Environment Chemical Computing Group (Labute, 2010), and the $\mathrm{N}$-amino terminus was sampled by Macromodel (Watts et al., 2014). From the numerous $\mathrm{N}$-amino termini and loop combinations obtained, we retained and minimized those in agreement with previously reported experimental data (Ji et al., 1997; Kennedy et al., 1997; Gigoux et al., 1998, 1999; Hadac et al., 1998; Ding et al., 2001, 2002; Giragossian and Mierke, 2001; Dong et al., 2009) and without substantial clashes.

We performed induced-fit docking of the CCK peptide in the best new model (out of several prioritized models), taking into account the mutational data and the reported potency/efficacy of peptide on the CCK1R mutants (Gigoux et al., 1998, 1999; Ding et al., 2002), intermolecular nuclear overhauser effects observed between CCK and the receptor (Kennedy et al., 1997; Giragossian and Mierke, 2001), as well as photoaffinity-labeling data (Ji et al., 1997; Hadac et al., 1998; Ding et al., 2001; Dong et al., 2009). These considerations are reviewed in Supplemental Table 1. The CCK1R-CCK peptide complex structure was subsequently used to dock SR146131.

The three-molecule complex (CCK1R + CCK peptide + SR146131) was then subjected to $1 \mu \mathrm{s}$ molecular dynamics simulation using Desmond (Bowers et al., 2006) after placing a 1-palmitoyl-2-oleoyl-glycero-3phosphocholine membrane system and addition of explicit solvent, TIP3P water system together with $\mathrm{Cl}^{-}$ions to neutralize the system. In addition, $0.15 \mathrm{M} \mathrm{NaCl}$ salt was also added to the system. The transmembrane segments annotated in the Uniprot entry for CCK1R were used for the placement of the 1-palmitoyl-2-oleoyl-glycero-3-phosphocholine bilayer. Twenty-six frames from the simulation were selected at even intervals of $40 \mathrm{~ns}$, starting at the initial frame, for docking of compound 12 using Glide (Schrödinger, Mannheim, Germany) (Friesner et al., 2004) without any constraints. A maximum of six poses was retained for each docking, and only those poses with a docking score better than -9.0 were subjected to binding mode analysis. For these poses, we observed a binding mode in which the acid group of compound 12 interacts with Arg 6.58 about $42 \%$ of the time and the same group interacts with Asn 6.55 for about $24 \%$ of the time. We also found that Tyr 4.63 interacts with the same acid group in a substantial number (33\%) of binding poses, either as sole partner or in addition to Asn 6.55. We also observed in a small number $(6 \%)$ of cases some interactions with Tyr 4.60 .

There are strong mutational data supporting the critical roles of Arg 6.58 and Asn 6.55 in SR146131 binding (Gouldson et al., 2000; Escrieut et al., 2002), so we decided to focus on these residues for 
further analysis. Supplemental Table 2 summarizes the data describing the functional impact of CCK1R mutations on SR146131 action and the ligand-receptor residue distances in our models. Two representative frames, each leading consistently to one of these two binding modes, were selected and used as starting points for docking of SR146131 and its analogs using Glide. In this study also, no constraints were employed for docking of these compounds to the CCK peptide-bound receptor structure. The top poses for docked SR146131 and its analogs were then submitted to Prime-molecular mechanics, the Born model and solvent accessibility method (mmGBSA) calculations (Sherman et al., 2006). This was used to refine ranking based on relative binding affinities $(\Delta G)$ and to evaluate structural changes on the receptor occurring after binding of this series of ligands, in both the presence and absence of the CCK peptide. Our models differ from all previously described molecular models of this receptor because they simultaneously accommodate binding of both the CCK peptide and a small-molecule ligand, in this study SR146131 and its analogs. This also may be helpful in understanding subtle differences in the functional behavior of different ligands.

Data Analysis and Statistics. All data were analyzed using Prism 6 or 7 (GraphPad Software, San Diego, CA). In all analyses, the data were unweighted, with the mean of replicates in each experiment considered as an individual point. Throughout the manuscript, data are presented as the mean of these values \pm S.E.M. with the number of replicates of independent experiments noted. Concentration-response data were analyzed using the three-parameter logistic equation (May et al., 2007), $\mathrm{E}=$ Bottom + (Top-Bottom) $[\mathrm{A}] /[\mathrm{A}]+\left[\mathrm{EC}_{50}\right]$, where Bottom represents the $\mathrm{E}$ value in the absence of ligand, Top represents the maximal stimulation in the presence of ligand, $[\mathrm{A}]$ is the molar concentration of ligand, and $\mathrm{EC}_{50}$ represents the molar concentration of ligand required to generate a response halfway between Top and Bottom. The competitive model was used for the analysis of the binding data, with data corrected for radioligand occupancy using the Cheng-Prusoff equation (Cheng and Prusoff, 1973). Differences in receptor binding and signaling parameters between various constructs were statistically evaluated using one-way analysis of variance with Dunnett's multiple comparison post-test, with $P<0.05$ considered to be statistically significant.

Radioligand equilibrium-binding curves with allosteric ligands were fitted to either a one-site inhibition mass action curve to determine inhibitor potency $\left(\mathrm{IC}_{50}\right)$ estimates, which were then converted to $\mathrm{K}_{\mathrm{i}}$ values, as appropriate, or an allosteric ternary complex model (eq. 1) to derive estimates of allosteric modulator affinity $\left(\mathrm{K}_{\mathrm{B}}\right)$ and cooperativity between the compound and radioligand $(\alpha)$; where $\alpha>1$ denotes positive cooperativity, $0<\alpha<1$ denotes negative cooperativity and $\alpha=1$ denotes neutral cooperativity (Ehlert, 1988; Christopoulos and Kenakin, 2002).

$$
E=\frac{B_{\max }[A]}{[A]+\left(K_{A}\right)\left(\frac{K_{B}+[B]}{K_{B}+\alpha[B]}\right)}
$$

where $K_{A}$ and $K_{B}$ represent the equilibrium dissociation constant of the radioligand and interacting ligand, respectively, and $[A]$ and $[B]$ denote their concentrations. $B_{\max }$ is the maximum number of binding sites on the cell. Dissociation kinetic data were fit to a one-phase exponential decay function to derive the apparent rate constant of dissociation $\left(\mathrm{K}_{\mathrm{off}}\right)$ in the absence or presence of each compound.

Functional studies for the interaction of orthosteric agonist and allosteric modulator were fitted to either a three-parameter logistic equation or an operational model of allosterism and agonism (Leach et al., 2007) (eq. 2) to derive estimates of affinity and combined binding/efficacy cooperativity:

$E=\frac{E_{m}\left(\tau_{A}[A]\left(K_{B}+\alpha \beta[B]\right)+\tau_{B}[B] K_{A}\right)^{n}}{\left([A] K_{B}+K_{A} K_{B}+K_{A}[B]+\alpha[A][B]\right)^{n}+\left(\tau_{A}[A]\left(K_{B}+\alpha \beta[B]+\tau_{B}[B] K_{A}\right)^{n}\right.}$ where $E_{m}$ is the maximum possible response for the system; [A] and $[B]$ are the concentration of the orthosteric agonist and allosteric modulator, respectively; $\tau_{\mathrm{A}}$ and $\tau_{\mathrm{B}}$ are the signaling efficacy of the respective ligands; $K_{A}$ and $K_{B}$ are the equilibrium dissociation constants for the respective ligands; $n$ is a transducer slope factor linking occupancy to response; $\alpha$ is the cooperativity factor (as described above); and $\beta$ is an empirical scaling factor describing the allosteric effect of the modulator on the orthosteric agonist signaling efficacy.

\section{Results}

Subtype Selectivity of SR14131 at Wild-Type CCK Receptors. Consistent with previous reports (Bignon et al., 1999; Gouldson et al., 2000), SR146131 displayed concentration-dependent agonism, stimulating maximal intracellular calcium responses in the CCK1R-bearing CHOCCK1R cells similar to that stimulated by natural CCK, while lacking agonism in the CCK2R-bearing CHO-CCK2R cell line over equivalent concentrations (Fig. 1B). This compound displayed high potency on CCK1R, with an $\mathrm{EC}_{50}$ of $43 \pm$ $2 \mathrm{pM}$ (mean \pm S.E.M., $n=6$ ), and substantial functional subtype selectivity over CCK2R (Fig. 1B).

Competition-binding studies using radiolabeled nonpeptidyl antagonist benzodiazepines (radioiodinated BDZ-1 and BDZ-2 for CCK1R and CCK2R, respectively), which bind to an intrahelical allosteric site (Cawston et al., 2012), were performed to assess the binding properties of SR146131 at both CCKR subtypes. SR146131 fully competed for binding of the ${ }^{125} \mathrm{I}-\mathrm{BDZ}-1$ at CCK1R, yielding a pK $\mathrm{p}$ of $8.30 \pm 0.09(n=6)$ using a competitive binding model (Fig. 1C), whereas it displayed incomplete inhibition $(\sim 50 \%)$ of the saturable binding of ${ }^{125}$ I-BDZ-2 at CCK2R $(n=4)$ (Fig. 1C), indicative of an allosteric mode of interaction between the two ligands. We recently demonstrated that similar behavior of another CCK receptor ligand was due to interactions across a receptor homodimer, and this is likely also true for the SR146131 compound (Desai et al., 2015c). To account for the allosteric nature of the binding, these data were subsequently analyzed using an allosteric ternary complex model (Christopoulos and Kenakin, 2002). The derived constant for SR146131 binding to the free CCK1R $\left(\mathrm{pK}_{\mathrm{B}}\right)$ was $8.24 \pm 0.08(n=5)$, and this was different from that at CCK2R (7.20 $\pm 0.17, n=4, P<0.05$, unpaired $t$ test). The cooperativity constants with the iodinated radioligand, $\log \alpha_{\mathrm{BDZ}-1}$ or $\log \alpha_{\mathrm{BDZ}-2}$, were also lower at CCK1R than at the CCK2R, respectively $(-1.22 \pm 0.10$ vs. $0.36 \pm 0.07, P<0.05$, unpaired $t$ test), indicating stronger negative cooperativity at the CCK1R compared with CCK2R.

\section{Allosteric Action of SR146131 at CHO-CCK1R Cells.} We examined possible PAM activity of SR146131 by measuring dose-response curves of both CCK and a phenylethyl ester analog of CCK (CCK-OPE), which is a partial agonist at the CCK1R, in CHO-CCK1R cells in the absence or presence of increasing concentrations of SR146131. These analyses confirmed that SR146131 is a PAM agonist because it potentiated both CCK- and CCK-OPE-dependent intracellular calcium mobilization (Fig. 2). The strong intrinsic agonism of SR146131 makes it difficult to apply operational models to quantify the degree of allosteric modulation because a maximal system response is reached at concentrations much lower than the predicted $\mathrm{pK}_{\mathrm{B}}$ of SR146131. In these interaction studies, low concentrations of SR146131 were used, and these 
A

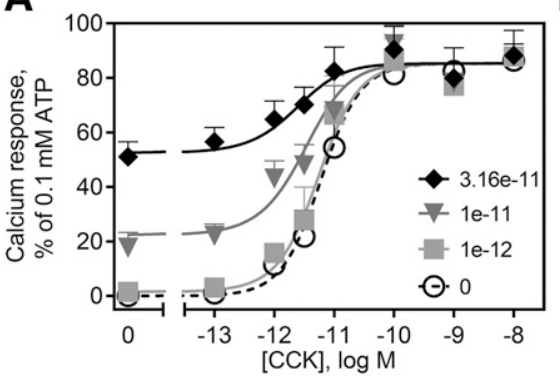

B

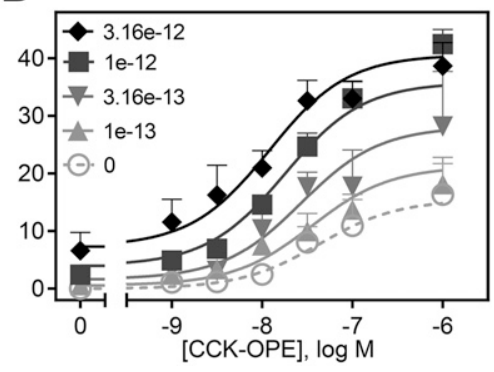

Fig. 2. Effect of increasing concentrations of SR146131 on CCK- and CCK-OPE-stimulated intracellular calcium responses. Shown are CCK-stimulated intracellular calcium dose-response curves on CHO-CCK1R cells in the absence or presence of increasing concentrations of SR146131. Data represent means \pm S.E.M. of data from $\operatorname{six}(0)$, four $\left(1 \mathrm{e}^{-12}\right)$, seven $\left(1 \mathrm{e}^{-11}\right)$, and nine $\left(3.16 \mathrm{e}^{-11}\right)$ experiments $(\mathrm{A})$. Shown also are the data generated from similar experiments performed using the partial agonist, CCK-OPE, on the same cells (B). Values are expressed as percentages of the intracellular calcium responses to maximum stimulation achieved by $0.1 \mathrm{mM}$ ATP. Data represent means \pm S.E.M. of data from seven $(0)$, eight $\left(1 \mathrm{e}^{-13}\right)$, four $\left(3.16 \mathrm{e}^{-13}\right)$, six $\left(1 \mathrm{e}^{-12}\right)$, and seven $\left(3.16 \mathrm{e}^{-12}\right)$ experiments. data were analyzed with the operational model of allosterism by fixing the $\mathrm{pK}_{\mathrm{B}}$ to the affinity of SR146131 defined in radioligand binding to yield composite (affinity and efficacy) cooperativity estimates. One caveat with fixing the $\mathrm{pK}_{\mathrm{B}}$ in this analysis is that the assay conditions for radioligand binding (equilibrium conditions and performed in membranes) and the functional assay (nonequilibrium and performed in whole cells) are not equivalent. Because the estimated $\mathrm{pK}_{\mathrm{B}}$ can differ under different experimental conditions, and $\mathrm{pK}_{\mathrm{B}}$ and $\alpha$ are linked in the operational analysis, under/overestimation of this value can influence the derived cooperativity estimates. Nonetheless, this analysis confirmed the high intrinsic agonist efficacy of SR146131 with consistent efficacies predicted from both data sets with operational $\log \tau_{\mathrm{B}}$ values of $2.41 \pm$ $0.06\left(\tau_{\mathrm{B}}=263\right)$ derived from the CCK interaction curve and $2.32 \pm 0.11\left(\tau_{\mathrm{B}}=209\right)$ from the CCK-OPE analysis. Consistent with visual observation of the data, cooperativity estimates from this analysis predict that SR146131 has greater cooperativity with CCK-OPE $\left[\log \alpha \beta_{\mathrm{CCK}-\mathrm{OPE}}=3.72 \pm 0.08\left(\alpha \beta_{\mathrm{CCK}-\mathrm{OPE}}=\right.\right.$ $5248)]$ than with $\mathrm{CCK}\left[\mathrm{Log} \alpha \beta_{\mathrm{CCK}}=2.75 \pm 0.25\left(\alpha \beta_{\mathrm{CCK}}=\right.\right.$ 562)].

SR146131 Function at CCK1R/CCK2R Chimeric Constructs. An experimental structure-activity relationship approach for the small-molecule ligand-binding site was used to interrogate the molecular basis of ligand-receptor and/or peptide interaction of SR146131. We have previously used this approach for molecular understanding of the action of other CCKR small-molecule antagonists (Cawston et al., 2012), and agonists (Harikumar et al., 2013; Desai et al., 2015c), using CCK1R/CCK2R constructs with exchanged intrahelical allosteric pocket residues between these receptors. Receptor density of stably expressed constructs and effect of mutation on BDZ and CCK ligand affinities have been previously reported (Cawston et al., 2012; Harikumar et al., 2013; Desai et al., 2015c). In the first series of studies, we used receptor constructs exchanging individual transmembrane domain (TM)2, TM3, TM6, and TM7 segments of CCK1R with the corresponding residues of CCK2R, as well as the converse constructs (Cawston et al., 2012; Harikumar et al., 2013; Desai et al., 2015b) (Fig. 3A; Table 1). In the case of the CCK1R-based chimeras, the TM7 construct [CCK1R $\mathrm{L} 7.39 \mathrm{H}$; all residue numbers are labeled according to the Ballesteros and Weinstein class A numbering scheme (Ballesteros and Weinstein, 1992)] showed greatest loss in ligand-induced intracellular calcium mobilization, followed by the TM2 construct (CCK1R N2.61T). Of note, at the TM3- and TM6-based chimeras (CCK1R T3.28V, T3.29S, and I6.51V, F6.52Y), there were small but statistically significant right shifts in $\mathrm{EC}_{50}$ values. Interestingly, of the converse CCK2R-based constructs with exchanged residues of CCK1R, only the TM7 and TM6 constructs exhibited a partial gain of function in response to SR146131 (Fig. 3B; Table 1).

Competition-binding experiments utilizing ${ }^{125} \mathrm{I}-\mathrm{BDZ}-1$ or ${ }^{125}$ I-BDZ-2 radioligands on the CCK receptor chimeras (Fig. $3 \mathrm{C}$; Table 1) demonstrated that, in the CCK1R-based
A
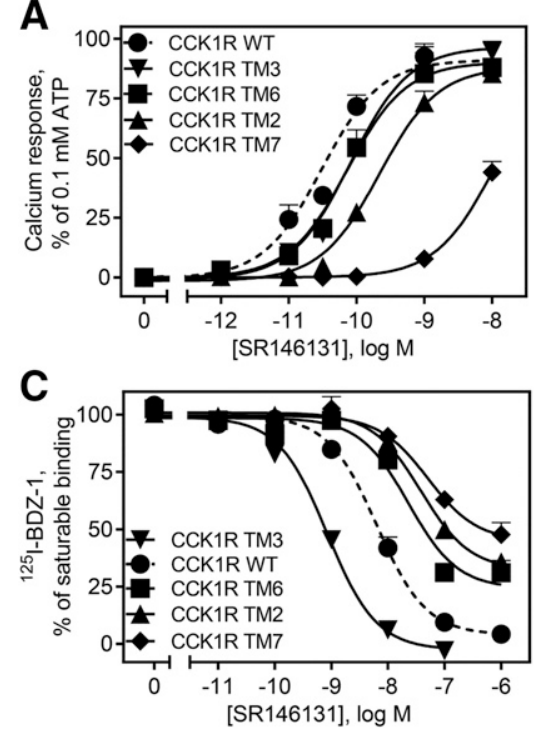

D
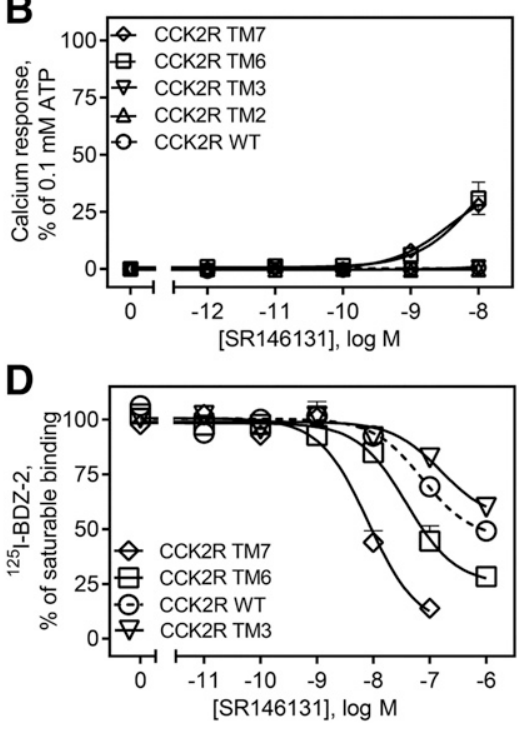

Fig. 3. Biologic activity and competition-binding studies of SR146131 at CCK1R- and CCK2R-based chimeric receptor constructs. The dose-response curves shown in this figure represent the ability of SR146131 to stimulate intracellular calcium responses in CHO cells bearing CCK1R (A) or CCK2R (B) WT and chimeric constructs. Data are represented in percentage responses relative to the maximum stimulation achieved by $0.1 \mathrm{mM}$ ATP. Shown also are the competition-binding curves for SR146131 at CCK1R-based (C) or CCK2R-based (D) TM chimeric constructs using the allosteric antagonist radioligands, ${ }^{125}$ I-BDZ-1 (for CCK1R) and ${ }^{125} \mathrm{I}-\mathrm{BDZ}-2$ (for CCK2R). Values represent percentages of maximal saturable binding that were observed in the absence of competitor. Nonsaturable binding was determined by using $1 \mu \mathrm{M}$ unlabeled BDZ-1 or BDZ-2, as appropriate for the radioligand. Data are expressed as means \pm S.E.M. of data from six experiments. 
TABLE 1

Pharmacology of SR146131 at WT and chimeric CCK1R and CCK2R

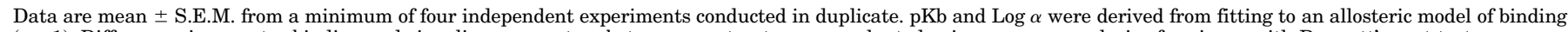
(eq. 1). Differences in receptor binding and signaling parameters between constructs were evaluated using one-way analysis of variance with Dunnett's post-test.

\begin{tabular}{|c|c|c|c|c|c|}
\hline Receptors & Receptor Abbreviations & $\mathrm{pIC}_{50}$ & $\mathrm{pEC}_{50}$ & $\mathrm{pK}_{\mathrm{b}}$ & $\log \alpha$ \\
\hline CCK1R WT & CCK1R & $8.10 \pm 0.09$ & $10.59 \pm 0.11$ & $8.20 \pm 0.08$ & $-1.20 \pm 0.10$ \\
\hline \multicolumn{6}{|l|}{$\begin{array}{l}\text { CCK1R-Based Chimeric } \\
\text { Receptors }^{a}\end{array}$} \\
\hline $\mathrm{N} 2.61 \mathrm{~T}$ & CCK1R TM2 & $6.85 \pm 0.04^{* *}$ & $9.70 \pm 0.11 * *$ & $7.30 \pm 0.11^{* *}$ & $-0.50 \pm 0.04^{* *}$ \\
\hline T3.28V, T3.29S & CCK1R TM3 & $9.13 \pm 0.07 * *$ & $10.13 \pm 0.09^{*}$ & $9.14 \pm 0.08^{* *}$ & $(\alpha \text { is close to } 0)^{* *}$ \\
\hline $\mathrm{I} 6.51 \mathrm{~V}, \mathrm{~F} 6.52 \mathrm{Y}$ & CCK1R TM6 & $7.26 \pm 0.04^{* *}$ & $10.12 \pm 0.06^{*}$ & $7.60 \pm 01^{* *}$ & $-0.64 \pm 0.08 * *$ \\
\hline $\mathrm{L} 7.39 \mathrm{H}$ & CCK1R TM7 & $6.33 \pm 0.20^{* *}$ & $8.00 \pm 0.10 * *$ & $7.24 \pm 0.11^{* *}$ & $-0.40 \pm 0.05^{* *}$ \\
\hline \multicolumn{6}{|l|}{$\begin{array}{l}\text { CCK2R-Based Chimeric } \\
\text { Receptors }\end{array}$} \\
\hline CCK2R WT & CCK2R & $6.23 \pm 0.13$ & NR & $7.20 \pm 0.17$ & $-0.36 \pm 0.07$ \\
\hline $\mathrm{T} 2.61 \mathrm{~N}$ & CCK2R TM2 & NDB & NR & NDB & NDB \\
\hline V3.28T, S3.29T & CCK2R TM3 & $5.94 \pm 0.09$ & NR & $7.06 \pm 0.28$ & $-0.24 \pm 0.08$ \\
\hline V6.51I, Y6.52F & CCK2R TM6 & $7.03 \pm 0.13^{* *}$ & $<6.0$ & $7.40 \pm 0.12$ & $-0.61 \pm 0.05^{*}$ \\
\hline $\mathrm{H} 7.39 \mathrm{~L}$ & CCK2R TM7 & $8.03 \pm 0.09 * *$ & $<6.0$ & $8.11 \pm 0.08^{* *}$ & $-1.11 \pm 0.06^{* *}$ \\
\hline
\end{tabular}

NDB, no detectable binding; NR, no response.

${ }^{a}$ Equilibrium binding was established using the CCK1R-selective small-molecule radioligand, ${ }^{125} \mathrm{I}-\mathrm{BDZ}-1$.

${ }^{b}$ Equilibrium binding was established using the CCK2R-selective small-molecule radioligand, ${ }^{125}$ I-BDZ-2.

$* P<0.05 ; * * P<0.01$, relative values compared with WT receptor.

chimeras, the TM7 construct had greatest impact on binding, followed by the TM2 and TM6 constructs, whereas the TM3 construct led to an increase in SR146131 $\mathrm{pIC}_{50}$ values. These data were analyzed with the operational allosteric model to yield values of $\mathrm{pK}_{\mathrm{B}}$ and $\log \alpha$ for the respective radioligands, ${ }^{125}$ I-BDZ-1 or ${ }^{125}$ I-BDZ-2, illustrating that the chimeras impacted both affinity and binding cooperativity (Table 1). The CCK2R-based constructs exhibited the converse increase or decrease in binding inhibition, although this was only statistically significant for the TM6 and TM7 chimeras (Fig. 3D; Table 1). For the TM6 chimera, the effect was primarily driven by increased negative cooperativity, whereas the TM7 chimera altered both affinity and cooperativity (Table 1). Of note, as reported in our previous studies (Cawston et al., 2012; Harikumar et al., 2013), the CCK2R TM2 chimera showed a complete loss of binding of ${ }^{125} \mathrm{I}$-BDZ-2 and hence could not be assessed. Overall, these data suggested a greater importance of residues constituting the CCK1R TM7 (Leu 7.39) and TM2 (Asn 2.61) constructs in docking of SR146131.
Modeling and Docking of SR146131 in the CCK1R Helical Bundle. SR146131 was initially docked into our previously reported model of the active conformation of the helical bundle of CCK1R (Harikumar et al., 2013) (Fig. 4). The best-fitting, most energetically favorable model predicted that the compound docked within the same intramembranous intrahelical pocket of the CCK1R previously predicted for GI181771X (Harikumar et al., 2013), with the thiazole ring of SR146131 and the benzodiazepine ring of GI181771X occupying the same space (Figs. 4 and 5). The main difference in these models is that GI181771X makes closer interactions with TM6, including two hydrogen bonds with Asn 6.55 and Arg 6.58 (Fig. 5B). The pocket occupied by SR146131 was formed by TM2, TM3, TM6, and TM7, with the indole ring oriented toward the extracellular region and the carboxylate group facing TM6 and making interactions with Asn 6.55. This model predicted that the residues Met 3.32, Val 3.36, and Trp 6.48 formed the steric bulk lining the bottom of the pocket. Residues Asn 2.61, Asn 2.65, and Tyr 7.43 formed a hydrogen-bonding network connecting

A

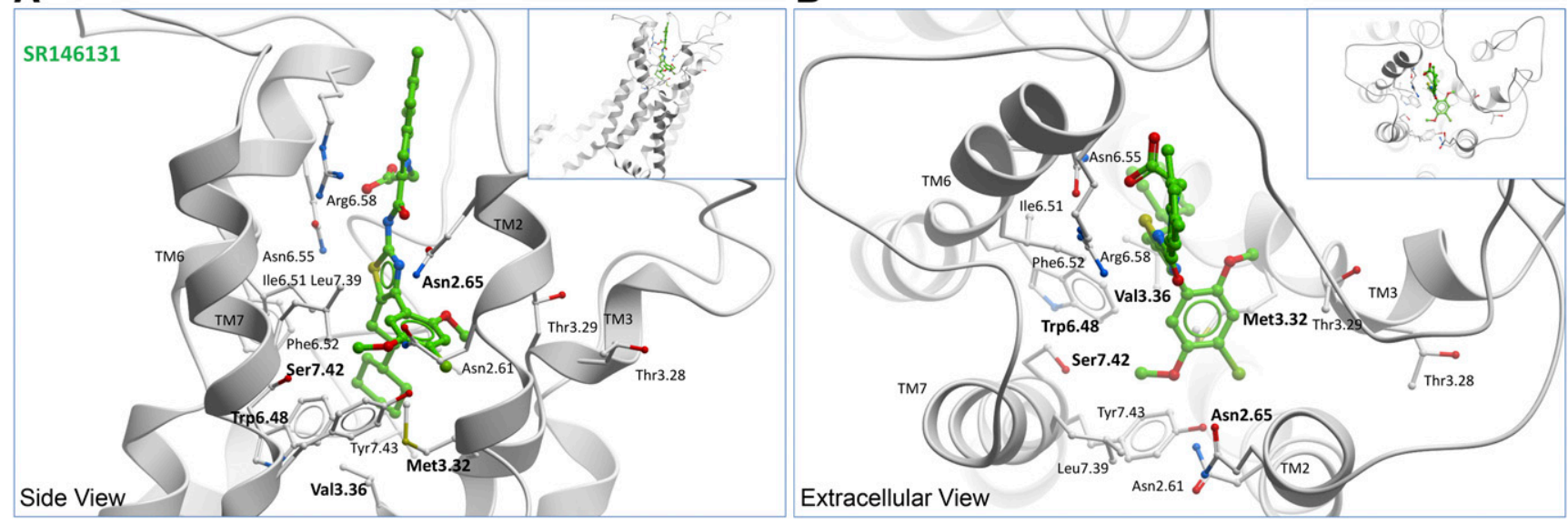

Fig. 4. Docking of SR146131 at the CCK1R. Shown is the best performing model for docked binding poses of SR146131 (green stick) to CCK1R (gray ribbon and stick) (A and B). The viewpoint in (A) is from the side within the membrane with TM1 cut away, and that in (B) is from above the membrane. Part of TM7 has been cut away for optimal viewing of the interactions. This model in Protein Data Bank format is provided in Supplemental Material file, S1. 

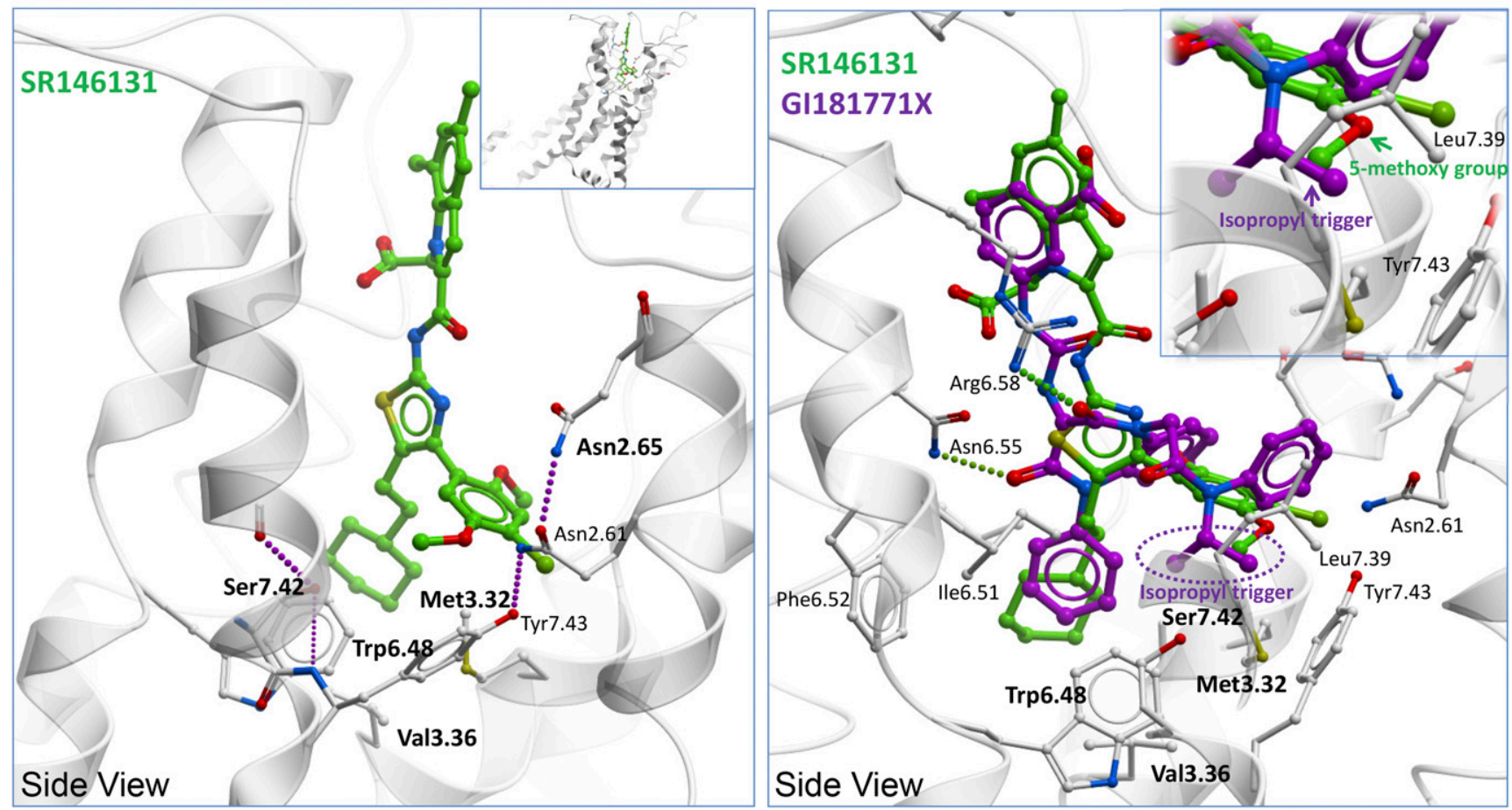

Fig. 5. Comparison between docking of SR146131 and GI181771X at the CCK1R. The key predicted interactions between SR146131 (green stick) and CCK1R that were studied by mutation are shown in light gray stick and ribbon in (A). Residues Trp 6.48 (W326A), Met 3.32 (M121A), and Val 3.36 (V125A) make contact area interaction of 17, 40, and $21 \AA^{2}$, with SR146131. Ser 7.42 (S359A) and Asn 2.65 (N102A) do not make contacts with SR146131, but make hydrogen bond interactions with other side chains (purple spheres), which are predicted to stabilize the conformation of the pocket. Ser 7.42 makes two hydrogen bonds with the backbone atoms of TM7, and Asn 2.65 is one part of a chain of hydrogen bonds with Asn 2.61 and Tyr 7.43. (B) shows a comparison of the docked binding pose of SR146131 (green stick) and GI181771X (purple stick): part of TM7 has been cut away for optimal viewing of the interactions. The pose of GI181771X is consistent with that reported in Harikumar et al. (2013) and docks in a similar region of the pocket as SR146131, with the thiazole ring of SR146131 and the benzodiazepine ring of GI181771X occupying the same space. The main differences are that GI181771X makes closer interactions with TM6, including two hydrogen bonds (small colored spheres) with Asn 6.55 and Arg 6.58. Inset: a close-up of the isopropyl group important for agonist activity of GI181771X. In SR146131, the carbon atom of the 5-methoxy group overlays this region of GI181771X. This model in Protein Data Bank format is provided in Supplemental Material files, S1 (SR146131) and S2 (GI181771X).

TM2 and TM7 (Figs. 4 and 5). Furthermore, Ser 7.42 formed two intrahelical hydrogen bonds with the backbone of TM7 (Figs. 4 and $5 \mathrm{~A}$ ).

As noted earlier, the agonist SR146131 and the closely related antagonist SR27897 differ by the presence of two methyl groups on the indole ring, an additional cyclohexylethyl group attached to the thiazol ring, and two methoxy groups on the phenyl ring of SR146131 (Gouldson et al., 2000). Because the two methyl groups on the indole ring are predicted to face the extracellular surface, they are unlikely to contribute any molecular interaction important for agonist activity. In contrast, the cyclohexylethyl group and the phenyl ring are predicted to bind deep inside the pocket (Figs. 4 and 5). According to this model, the cyclohexylethyl group is predicted to point toward the pocket formed by TM3, TM5, and TM6 to make key interactions with Val 3.36 (contact area $21 \AA^{2}$ ), Trp $6.48\left(17 \AA^{2}\right.$ ), and Phe 6.52, and the phenyl ring is accommodated in the hydrophobic pocket formed by TM2, TM3, and TM7. This model predicts important interactions of the methoxy groups on the phenyl ring with residues Thr 3.29 and Leu 7.39 that were important for SR146131 function, as observed in the CCK1R/CCK2R chimeric receptor data. The model also predicts that the 2-methoxy group interacts with Thr 3.29, Tyr 3.30, and Met $3.32\left(40 \AA^{2}\right)$, and the 5-methoxy group can interact with Leu 7.39, Ser 7.42, Asn 2.61, and Met 3.32 (Figs. 4 and 5A).
Characterization of Receptor Mutants Predicted To Be Important for SR146131 Activity. Based on the proposed binding hypothesis by docking of SR146131, we identified additional receptor residues that were predicted to be in spatial proximity to the ligand, and thus could have functional importance. Of these, we used alanine-replacement mutants for Met 3.32, Val 3.36, and Trp 6.48 that were used in an earlier study [Bmax, pmol/mg: CCK1R wild-type (WT), $5.0 \pm$ 1.0; M3.32A, $151 \pm 63$; V3.36A, $0.9 \pm 0.4$; W6.48A, $0.8 \pm 0.2]$ (Desai et al., 2015c), and also generated an alanine-replacement mutant for Ser 7.42 (Bmax, pmol/mg: $82.6 \pm 12.0)$. Biologic activity and binding parameters for SR146131 at these CCK1R constructs are reflected in Fig. 6 and Table 2. Because of the differences in levels of receptor construct expression, we include $\log \tau_{\mathrm{B}}$ values normalized to levels of cell surface binding, Bmax, in Table 2 to reflect intrinsic efficacy of SR146131 at each of the receptor constructs relative to that at WT CCK1R. This difference was statistically significant for three of the constructs, with efficacy decreased for M3.32A and increased for V3.36A, and S6.48A.

Functional Effects of Modification of SR146131. In an attempt to reduce the agonist activity of SR146131 while still maintaining its PAM activity, we used structurally related compounds that were available from Sanofi with modifications on the phenyl ring that was predicted to be located at an equivalent position to the isopropyl group of GI181771X 


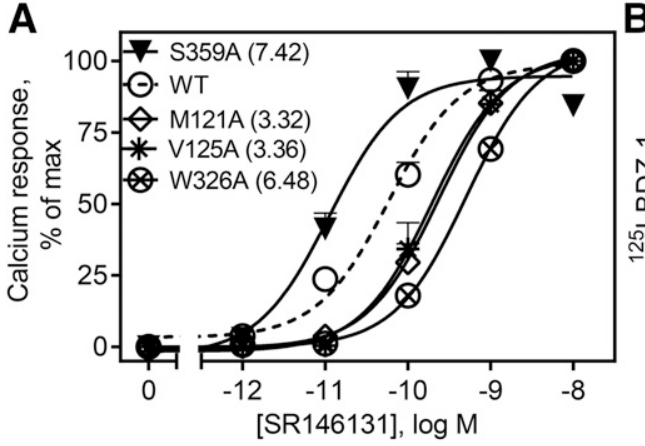

(Table 3). Of these, compounds with appreciable structural differences in this part of the molecule that retained the PAM activity included compound 2 (methyl group), compound 4 (hydrogen), and compound 12 (ethyl group) (Fig. 7). These compounds showed 1.3-, 1.3-, and 1.2-fold lower potencies than SR146131, respectively, at the CCK1R WT (Table 3). Using ${ }^{125} \mathrm{I}-\mathrm{BDZ}-1$ as radiolabel, $\mathrm{IC}_{50}$ values of compounds 2,4 , and 12 were determined to be 3.65-, 5.0-, and 32.0 times lower than SR146131, respectively $\left(\mathrm{pK}_{\mathrm{i}}\right.$ values: $7.65 \pm 0.03$, compound 2 ; $7.52 \pm 0.04$, compound $4 ; 6.70 \pm 0.02$, compound $12, n=3$ ). Of note, the $\mathrm{pIC}_{50}$ of compound 2 was different from compound $12(P<0.05$; one-way analysis of variance, Dunnett's posttest). Application of the operational model for allosterism revealed that compound 2 and compound 4 had markedly diminished efficacy and cooperativity (compound 4 exhibiting no PAM activity), with greatest impact seen with compound 4 (compound 2, $\log \tau_{\mathrm{B}}=0.42, \log \alpha \beta=0.76$; compound 4, $\log \tau_{\mathrm{B}}=0.02, \log \alpha \beta=0.06$ ) (Fig. 8). Compound 12 was unique among these SR146131 analogs in that it retained efficacy and positive allosteric modulatory activity on CCK action that was similar to that of the parental compound (Fig. $9 \mathrm{~A}$ ), with a $\log \tau_{\mathrm{B}}$ value of $2.27 \pm 0.04$ and $\log \alpha \beta$ of $2.41 \pm 0.19$ $(\alpha \beta=257)$. Of note, this effect was also seen on cells expressing 11-fold lower CCK1R WT on their surface, $\log \tau_{\mathrm{B}}=1.17$ and $\log \alpha \beta=1.41$. Interestingly, like SR146131, compound 12 exhibited stronger PAM effect when using the partial agonist, CCK-OPE, as the orthosteric ligand (Fig. 9B). The quantitative parameters for this effect were $\log \alpha \beta=4.18 \pm 0.08$ and $\log \tau_{\mathrm{B}}=2.28 \pm 0.36$.

Enhanced Molecular Model for Docking of SR146131 to the CCK1R Holoreceptor. Using the available structure-function data and the new model derived from the ligand-bound orexin-1 receptor as template, we have generated several refined models of CCK1R by loop refinement, molecular dynamic simulations, and ensemble dockings.
Based on this, we were able to develop a model that allowed the simultaneous occupation of both CCK peptide and SR146131 or its analogs. We believe that this is the first reported CCK1R model with natural peptide ligand and a small-molecule modulator ligand bound simultaneously. In these models, the two ligands reside adjacent to each other, with predicted ligand-ligand interactions that likely contribute to some of the biologic behavior of the tested small molecules (Figs. 10 and 11). The synergistic effect of the peptide and the small-molecule ligand binding could be deciphered by sampling of the extracellular domain and the loops, molecular dynamics simulations, and mmGBSA calculations, which had not been previously exploited for this receptor. We have used these new models to guide interpretation of the cooperativity of both CCK peptide and the smallmolecule ligands. Two of these models, corresponding to docking poses of SR146131 and analogs into two representative frames from the molecular dynamics simulations mentioned in the Materials and Methods section, are discussed below.

Molecular Modeling and Binding Hypothesis for SR146131 and Its Analogs. Initial models of the ternary complex among CCK1R, CCK peptide, and SR146131 were further subjected to molecular dynamics simulations to generate refined binding site and CCK peptide conformations for the 13 structurally related analogs of SR146131 discussed above. Two putative binding modes for SR146131 were observed from the molecular dynamics simulations with about the same frequency. Changes in binding mode seem driven by slight structural variations in the receptor and CCK peptide over the course of the molecular dynamics simulations. We decided to investigate both binding modes in more detail and performed docking studies for all 14 compounds in both pocket conformations using Glide (Schrödinger) (Friesner et al., 2004). Top poses for these active compounds were consistent

TABLE 2

SR146131-binding parameters and potency for CCK1R-based single-mutant constructs

\begin{tabular}{llcrr}
\hline CCK1R-Based Chimeric Receptors & \multirow{2}{*}{ Receptor Abbreviations } & \multicolumn{2}{c}{$\mathrm{pIC}_{50}$} & \multicolumn{2}{c}{$\mathrm{pEC}_{50}$} & \begin{tabular}{c} 
Log $\tau_{\mathrm{B}}$ Corrected for $_{\mathrm{Bmax}^{a}}$ \\
\hline CCK1R WT
\end{tabular} CCK1R $_{\text {M3.32A }}$ & $8.08 \pm 0.10(n=10)$ & $10.34 \pm 0.11(n=8)$ & $0.76 \pm 0.09$ \\
V3.36A & CCK1R M121A & $9.61 \pm 0.15^{* *}(n=5)$ & $9.90 \pm 0.16^{*}(n=4)$ & $-1.62 \pm 0.06^{* * * * *}$ \\
W6.48A & CCK1R V125A & $7.97 \pm 0.13(n=6)$ & $9.60 \pm 0.05^{* *}(n=5)$ & $1.83 \pm 0.11^{* * * * *}$ \\
S7.42A & CCK1R W326A & $8.14 \pm 0.08(n=6)$ & $9.30 \pm 0.06^{* *}(n=4)$ & $1.44 \pm 0.04^{* * * *}$ \\
\hline
\end{tabular}

${ }^{a}$ Intrinsic efficacy of SR146131 in each of the cell lines, compared with that in WT CCK1R.

$* P<0.05$; ** $P<0.01$; **** $P<0.0001$, relative values compared with WT CCK1 receptor using one-way analysis of variance, Dunnett's post-test. 
TABLE 3

Substitutions at "R" in the compounds structurally related to SR146131

The series is arranged in descending order of potency. $\mathrm{pEC}_{50}$ values represent means \pm S.E.M.

\begin{tabular}{|c|c|c|c|c|c|c|c|c|}
\hline Positions & 2 & 3 & 4 & 5 & 6 & $\begin{array}{c}\text { Indole } \\
\text { Substitutions }\end{array}$ & $\mathrm{pEC}_{50}$ & Emax \\
\hline SR 146131 & Methoxy & & $\mathrm{Cl}$ & Methoxy & & 5,7-dimethyl & $10.50 \pm 0.20$ & \\
\hline 10 & Methoxy & & $\mathrm{Me}$ & & Methoxy & & $10.17 \pm 0.17(n=5)$ & $124.4 \pm 7.7$ \\
\hline 12 & Methoxy & & Methoxy & Ethyl & & 5-methyl & $8.81 \pm 0.12^{*}(n=5)$ & $103.7 \pm 4.6$ \\
\hline 9 & Methoxy & & $\mathrm{Me}$ & MethoxyMe & & & $8.77 \pm 0.16^{*}(n=5)$ & $104.5 \pm 5.7$ \\
\hline 7 & $\mathrm{Me}$ & & $\mathrm{Me}$ & Methoxy & & & $8.60 \pm 0.13^{*}(n=5)$ & $97.9 \pm 1.1$ \\
\hline 3 & Methoxy & & $\mathrm{Cl}$ & $\mathrm{Cl}$ & & & $8.30 \pm 0.10 *(n=5)$ & $113.2 \pm 6.0$ \\
\hline 11 & Methoxy & & Methoxy & $\mathrm{Me}$ & & 5,7-dimethyl & $8.30 \pm 0.10 *(n=5)$ & $102.9 \pm 4.4$ \\
\hline 4 & Methoxy & & Methoxy & & & & $8.22 \pm 0.14 *(n=5)$ & $92.5 \pm 5.4$ \\
\hline 2 & Methoxy & & $\mathrm{Me}$ & $\mathrm{Me}$ & & & $8.17 \pm 0.14 *(n=4)$ & $94.7 \pm 6.4$ \\
\hline 8 & Methoxy & & dioxalane & & & & $7.72 \pm 0.18^{*}(n=5)$ & $73.7 \pm 9.4$ \\
\hline 1 & Methoxy & & $\mathrm{Me}$ & $\mathrm{Cl}$ & & & $7.58 \pm 0.13^{*}(n=4)$ & $92.0 \pm 10.0$ \\
\hline 13 & $\mathrm{Cl}$ & & & & & & $7.28 \pm 0.16^{*}(n=5)$ & $61.6 \pm 8.5^{* *}$ \\
\hline 5 & Methoxy & Methoxy & $\mathrm{Me}$ & & & & $7.02 \pm 0.23^{*}(n=3)$ & $25.6 \pm 4.5^{* * *}$ \\
\hline 6 & $\mathrm{Me}$ & $\mathrm{Me}$ & Methoxy & & & & $6.85 \pm 0.18^{*}(n=3)$ & $19.0 \pm 8.5^{* * *}$ \\
\hline
\end{tabular}

${ }^{*} P<0.05 ;{ }^{* * P}<0.01 ; * * * P<0.001$, calculated using one-way analysis of variance, Dunnett's post-test.

with the original SR146131 putative binding mode in both cases with highly negative docking scores, reinforcing our confidence in the new model. For all 14 ligands studied and in both binding modes, the thiazole ring of the small-molecule ligand exhibits pi stacking with the tryptophan (Trp 30) of the CCK peptide, indicating potential direct interaction between the endogenous agonist and the small-molecule ligands as a component of their cooperativity. Figures 10 and 11 exemplify the two putative binding modes for docked compound 12 by itself and in comparison with docked SR146131 and are discussed in detail below. General observations are also given for other representative analogs studied in this work.

Binding mode 1 (Fig. 10A), in which the acid group of the compounds is involved in a salt bridge with Arg 6.58, gave the best docking scores and most consistent poses for all 14 compounds. This binding mode is in reasonable agreement with most of the mutational data reported to date that have impact on the binding of SR146131 to CCK1R. This involves loss of activity of SR146131 at receptor constructs with mutations such as C94L (Cys 2.57 to Leu), F97A (Phe 2.60 to Ala), G122L (Gly 3.33 to Leu), V125A (Val 3.36 to Ala), F198A [part of extracellular domain (ECL)2], I329A(F) (Ile 6.51 to Ala or Phe), R336A(D)(M) (Arg 6.58 to Ala or Asp or Met), I352A (Ile 7.35 to Ala), and L356A (Leu 7.39 to Ala). All of these residues are in the close vicinity of this ligand's proposed binding site (see Fig. 10 for orientations of these residues, except Gly 3.33).

However, the effect of the N333A (Asn 6.55 to Ala) mutation to the loss of binding affinity of SR146131 to CCK1R could not be easily rationalized by this binding hypothesis, although the docked compounds are within $6.4 \AA$ of Asn 6.55. Additional induced effects and thus stronger interactions of Asn 6.55 with the ligand may be possible because this residue is within TM6, known to exhibit considerable movement and to play important functional roles in many GPCRs (Rasmussen et al., 2011; Latorraca et al., 2017).

Binding mode 2 (Fig. 10B), when compared with binding mode 1 , shows a flipped orientation of the indole moiety. In this case, the acid group of the ligand is involved in a hydrogen bond with the side chain of Asn 6.55, whereas the indole scaffold has a stacking interaction with the side chain of Arg 6.58. In this binding mode (Fig. 11B), we observe an additional difference between docked SR146131 and compound 12 of approximately $1 \AA$ shift of the indole scaffold due to the additional methyl group at position 7 and the proximity of the side chain of Asn 6.55. This binding mode also corresponds to good docking scores, although less negative, and increased variability in docking poses. Due to these reasons, we

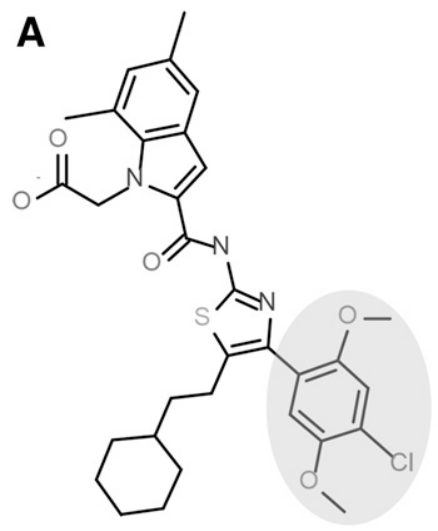

SR146131

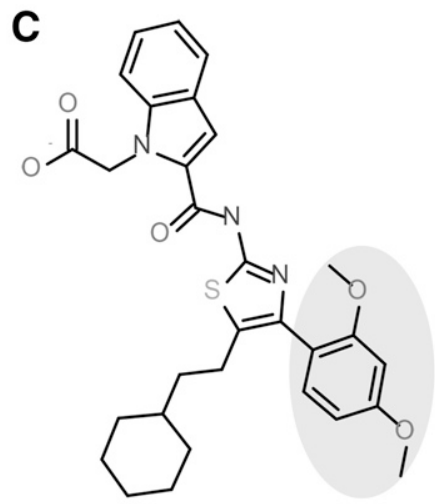

\section{Compound 4}

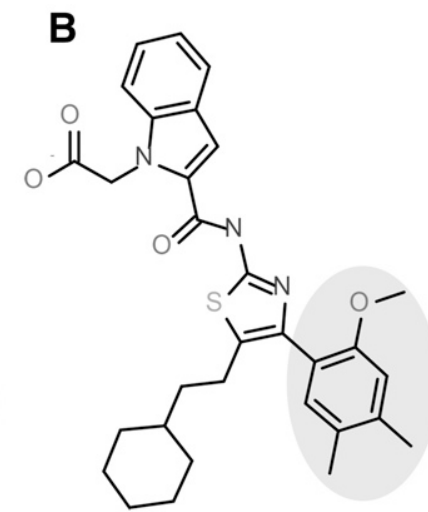

Compound 2

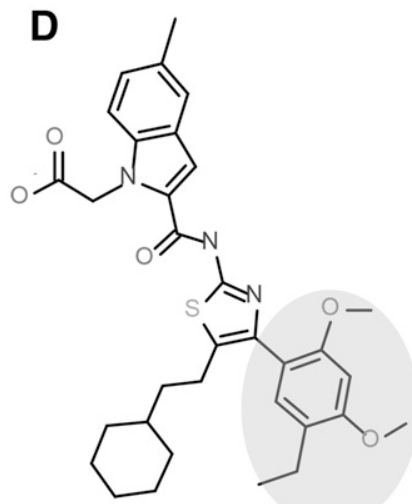

Compound 12
Fig. 7. Structures of compounds 2, 4, and 12. Shown is the region of SR146141 that is analogous to the isopropyl group of GI181771X, which has been shown to be critical for its agonist activity, shaded in gray (A), along with structures of compounds 2 (B), 4 (C), and 12 (D). 
A

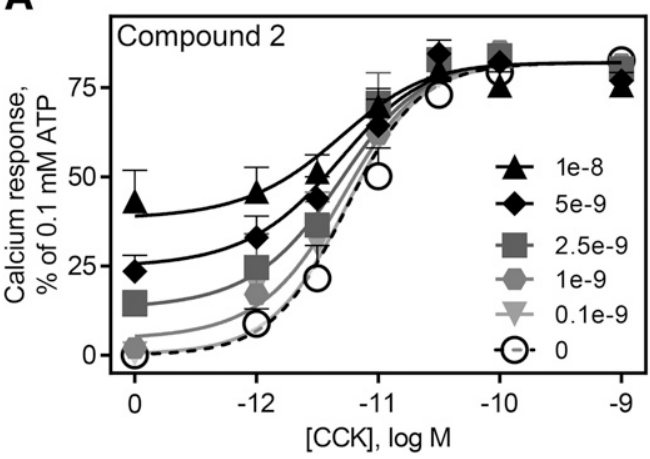

B

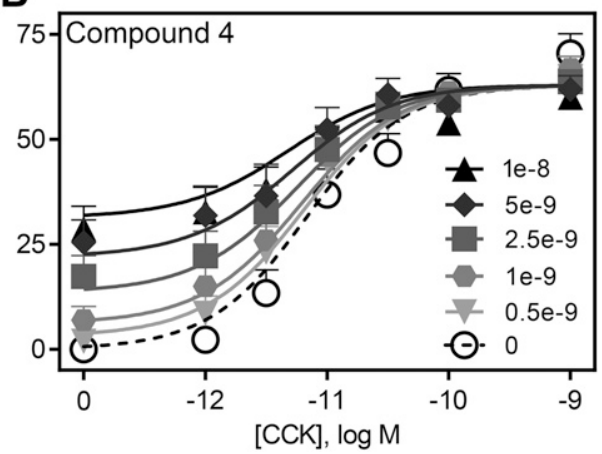

Fig. 8. Effect of increasing concentrations of compounds 2 and 4 on CCKstimulated intracellular calcium responses. Shown are CCK-stimulated intracellular calcium dose-response curves on $\mathrm{CHO}-\mathrm{CCK} 1 \mathrm{R}$ cells in the absence or presence of increasing concentrations of compound 2 (A), and compound 4 (B), with values expressed as percentages of the response to maximum stimulation achieved by $0.1 \mathrm{mM}$ ATP. Data represent means \pm S.E.M. of data from five $\left(10 \mathrm{e}^{-9}, 5 \mathrm{e}^{-9}, 2.5 \mathrm{e}^{-9}, 1 \mathrm{e}^{-9}\right)$, three $\left(0.1 \mathrm{e}^{-9}\right)$, and six $(0)$ experiments (A), and nine experiments for all doses (B). prioritized binding mode 1 and the Prime-mmGBSA calculations discussed in the next section focus on binding mode 1 .

The lower half of the compounds, below the amide linker, exhibits similar orientations and interactions in both binding modes, independent of the orientation of the indole moiety: Cys 2.57, Val 3.36, Trp 6.48, and Ile 6.51 are involved in hydrophobic interactions with the cyclohexyl moiety, in agreement with both new mutational data reported in this study and historical experimental data (Gouldson et al., 2000). Detailed analysis of compound 12 binding modes 1 and 2 predicts that Asn 2.61, Met 3.32, and Ser 7.42 form interactions with both the cyclohexyl and the substituted benzyl group. Phe 2.60, Asn 2.65, Phe 198, Leu 7.39, and Tyr 7.43 delimit the substituted benzene ring binding region. In addition, this new model sheds light on one potentially interesting aspect of PAM regulation: in both binding modes 1 and 2, the substituted benzene of compound 12 is near CCK peptide residue Trp 30 , where they show stacking interactions (in addition to the stacking of the thiazole ring to Trp 30 within the CCK peptide observed for all representatives) (Fig. 10). Differences were observed for the interactions of benzene ring substituents to the receptor in the two models. Particularly to compound 12 versus SR146131, the substitution in position 5 (ethyl vs. methoxy groups) led to differences in interaction energies with Leu 7.39 and Ser 7.42. Similar changes were seen in the interaction with Asn 2.65 with substitutions at position 4 (Cl vs. methoxy).

Prime-mmGBSA Calculations for SR146131 and Its Analogs in Binding Mode 1. In an attempt to better understand the effect of substitutions around SR146131, we ran Prime-mmGBSA induced-fit calculations for all compounds docked in CCK1R with previously described binding mode 1 , in both the presence and absence of CCK peptide. Figure 12 highlights the differences in CCK1R conformations between bound compound 10 (pure allosteric agonist) and compound 12 and SR146131 (PAM agonists), as a result of Prime-mmGBSA calculations in the presence of CCK peptide. Within TM7, changes in Tyr 7.43 seem to have a downstream impact on Thr 7.44 and Ser 7.45 (not shown for clarity), suggesting a potential signal transduction mechanism through TM7. Further changes down TM7 were not observed, probably due to the $10 \AA$ radius limit selected for the Prime-mmGBSA induced-fit calculation. The most striking differences were observed for TM2, probably driven by the combination of changes relative to Asn 2.61 and Asn 2.65, and to a lesser extent Pro 2.64. The top of TM2 was pushed the most in the complex with compound 10 and the least in the complex with SR146131. This shift in TM2 had impact on ECL1 position. In addition, Phe 198, which is present in ECL2, was also slightly shifted as well as the loop overall. ECL1 and ECL2 participate in CCK peptide binding, and the predicted differences in ligand conformations would provide a different environment for the peptide, leading to potential changes in affinity, residence time, and consequent functional effects.

Figure 13 summarizes the calculated $\Delta \mathrm{G}$ for each ligand with or without CCK peptide present in the binding site. In general, we see a decrease in $\Delta \mathrm{G}$ with or without CCK peptide,
A

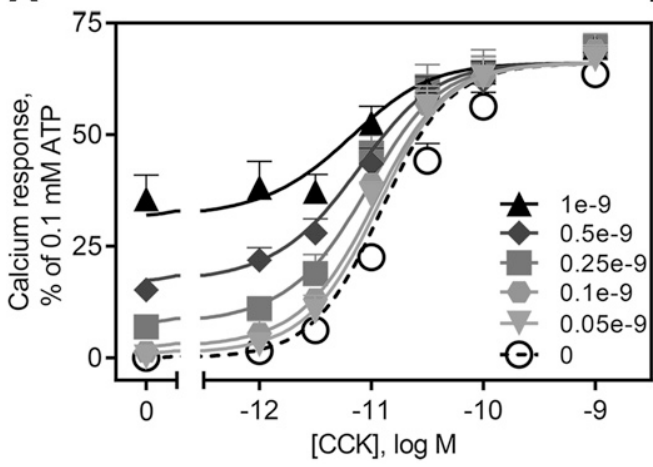

B

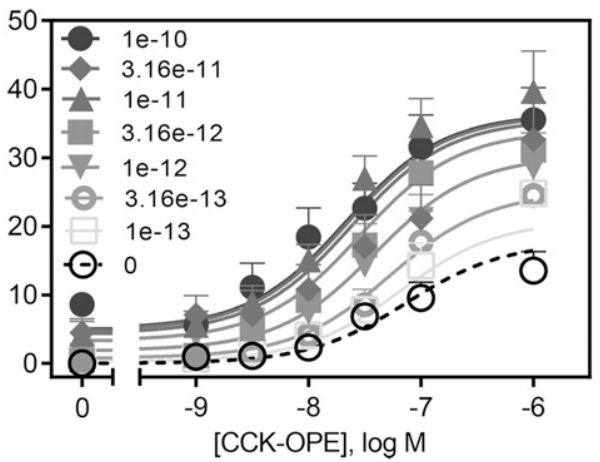

Fig. 9. Effect of increasing concentrations of compound 12 on CCK- and CCK-OPEstimulated calcium responses. Shown are effects of increasing concentrations of compound 12 on CCK-stimulated intracellular calcium dose-response curves in $\mathrm{CHO}$ cells stably expressing CCK1R at $19.2 \pm 2.5$ $\mathrm{pmol} / \mathrm{mg}$ (A). Shown also are the data generated from analogous experiments performed using the partial agonist, CCK-OPE (B). Values are expressed as percentages of the response to maximum stimulation achieved by $0.1 \mathrm{mM}$ ATP. Data represent means \pm S.E.M. of data from $11\left(0,0.5 \mathrm{e}^{-9}\right.$, $\left.1 \mathrm{e}^{-9}\right), 9\left(0.25 \mathrm{e}^{-9}\right)$ and $12\left(0.05 \mathrm{e}^{-9}, 0.1 \mathrm{e}^{-9}\right)$ experiments $(\mathrm{A})$, and $6\left(3.16 \mathrm{e}^{-11}\right), 9\left(1 \mathrm{e}^{-12}\right.$, $\left.3.16 \mathrm{e}^{-12}, 1 \mathrm{e}^{-11}, 1 \mathrm{e}^{-10}\right), 7\left(1 \mathrm{e}^{-13}, 3.16 \mathrm{e}^{-13}\right)$, and $14(0)$ experiments (B). 
A

B

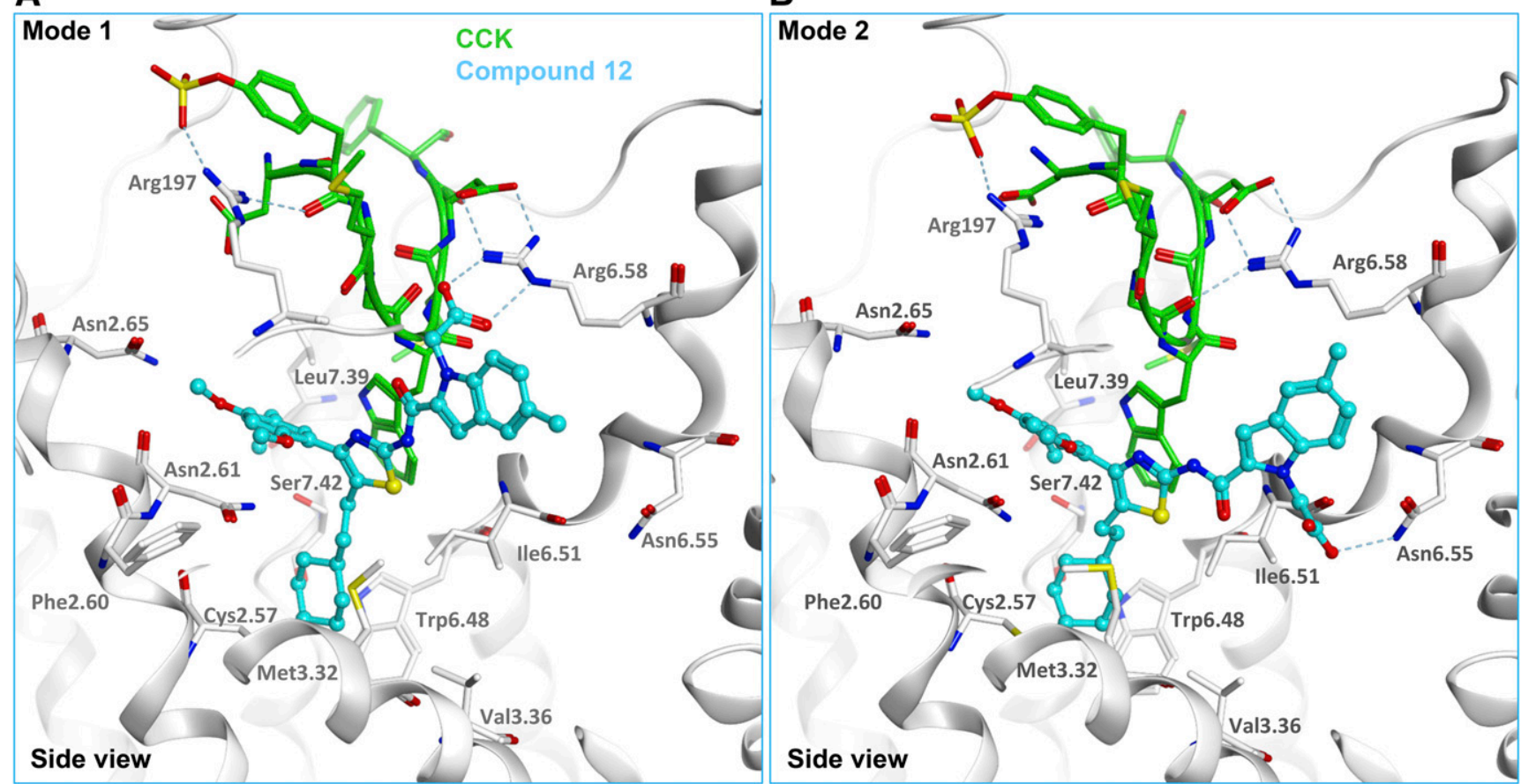

Fig. 10. Model of CCK and compound 12 bound to the CCK1R. Shown is a representation of the ternary structures of the CCK1R (in light gray) docked with the CCK peptide (in green) and the PAM compound 12 (in cyan) for binding mode 1(A), where the acid group is involved in a salt bridge with Arg 6.58, and for binding mode $2(\mathrm{~B})$, where the acid group is involved in a H-bond with Asn 6.55 side chain. In both cases, potential H-bonds are represented with light blue dashed lines. Some helices and residues were clipped for clarity. These models in Protein Data Bank format are provided in Supplemental Material files, S3 (mode 1) and S4 (mode 2).

which is in correlation with decrease in potency as determined experimentally (see Table 3 ). We observe a more favorable (more negative) $\Delta G$ in the presence of CCK peptide for approximately half of the compounds, including SR146131 and compound 12, both with PAM agonist properties. Compound 10 and compound 4 , both experimentally shown to lack PAM activity, were the only two compounds with a more favorable $\Delta \mathrm{G}$ in absence of CCK peptide. The other compounds did not display any large change in $\Delta \mathrm{G}$ (Fig. 13). This provides qualitative insights into the complexes with various compounds; however, a more quantitative assessment of binding affinities and functional effects would require more detailed calculations with large-scale conformational sampling using molecular dynamics simulations and replica exchange methods.

\section{Discussion}

CCK induces satiety by acting on the CCK1R present on axonal projections of vagal afferent neurons present in the gut (Smith et al., 1981, 1985; Li and Owyang, 1994). This has provided a rationale for attempts to develop agonists of this receptor as noncaloric satiety agents, potentially useful for the management of obesity (Aquino et al., 1996; Berger et al., 2008; Elliott et al., 2010). Several synthetic agonists have been developed for this purpose; however, none has gained regulatory approval for this application. This is mainly due to failure to reach the desired efficacy in humans (Jordan et al., 2008), with more potent analogs often possessing side effects, and longer duration agonists avoided due to concerns about potential trophic effects (Hoshi and Logsdon, 1993; Smith and Solomon, 2014).
We have proposed a possible alternative strategy to circumvent the concerns about side effects and possible toxicity of a highly potent full agonist, by working to develop CCK1R ligands with pure PAM activity for CCK, without possessing any intrinsic agonist activity (Desai et al., 2015a,b, 2016a,b, 2017; Miller and Desai, 2016). In this approach, the modulator would be expected to remain silent in the absence of CCK, but then to act as a PAM to enhance the activity of CCK at the CCK1R only during the brief interval after meals when the hormone is released, thereby minimizing on-target side effects and possible toxicity. It also reduces the likelihood of desensitization. Indeed, this strategy has been successfully used at other receptors, including the G protein-coupled calciumsensing receptor and the ionotropic GABA-A receptor (Barker et al., 1986; Nemeth et al., 2004). In addition to the usefulness of high-throughput screening for such candidates, we have also postulated an alternate strategy in which the determinants of agonist activity of an existing synthetic agonist can be removed, while retaining the components for causing a conformational change of the receptor to stabilize G protein association by reducing the energy barrier to achieve the active state (Desai et al., 2015b).

In the current study, we demonstrate that SR146131, which is a CCK1R-specific, highly potent agonist (Bignon et al., 1999), also possesses PAM activity, hence categorizing it as a PAM agonist. Thus, this compound is distinct from the previously characterized 1,5-benzodiazepine agonist, GI181771X (Desai et al., 2015b). Subsequent use of CCK1RCCK2R TM chimeric constructs enabled development of a molecular model for compound binding to the CCK1R, where SR146131 was accommodated in the same agonist pocket as 


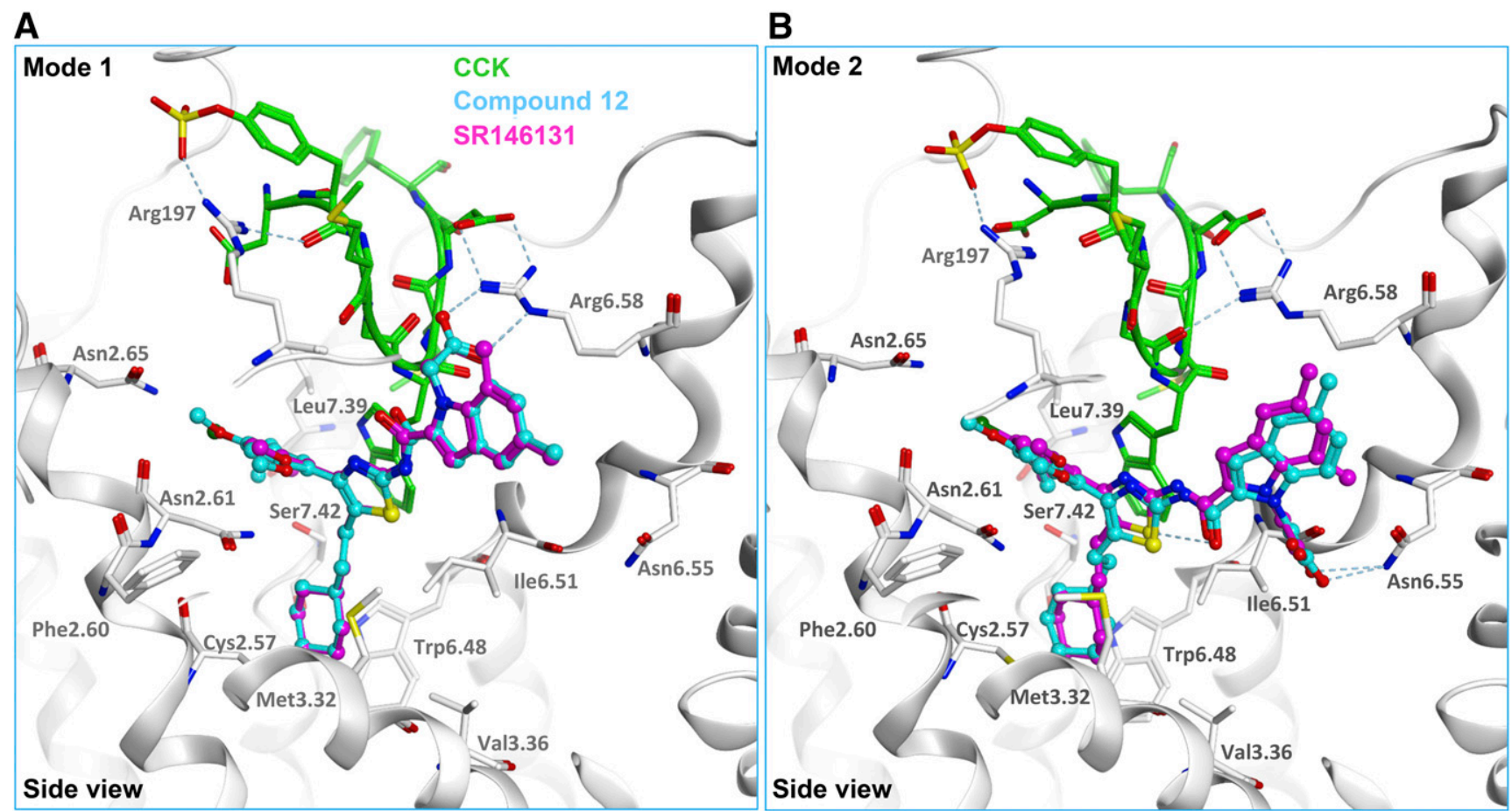

Fig. 11. Comparison between the docking of compound 12 and SR146131 at CCK1R. A comparison of receptor-interacting residues in the docked binding poses of SR146131 (purple stick) and compound 12 (cyan stick) is shown for binding modes 1(A) and 2 (B). CCK is shown in light green. The differences between both compounds in substitutions on the benzene ring, postulated to be important for its agonist activity, led to differences in interaction with Leu 7.39 and Ser 7.42, as well as Asn 2.65. Some helices and residues were clipped for clarity. These models in Protein Data Bank format are provided in Supplemental Material files, S3 (mode 1, compound 12), S4 (mode 2, compound 12), S5 (mode 1, SR146131), and S6 (mode 2, SR146131).

that predicted for GI181771X. Of note, this modeling predicted different docking poses of SR146131 compared with previous models that were developed prior to our current understanding of class A GPCR crystal structures (Gouldson et al., 2000).

Because the receptor conformation elucidated in initial docking of SR146131 was not substantially different from that achieved using GI181771X, we hypothesized that the region critical for agonist activity could also be in analogous parts of both ligands. In the case of GI181771X, the isopropyl group interacts with Leu 7.39, which is a major determinant of the agonist activity (Harikumar et al., 2013). The portion of SR146131 that was predicted to be adjacent to this part of the receptor is the phenyl ring. Indeed, although that ring is present in the structurally related antagonist, SR27897, it does not include the two methoxy groups present on the agonist. For this reason, there was a focus of interest in attempts to reduce agonist activity of the parent PAM agonist.

We examined 13 compounds that were structurally similar or related to SR146131 with substitutions on the phenyl ring and observed that the intrinsic agonist activity was reduced by varying degrees. We selected compounds 2,4 , and 12 from the series based on single-point PAM assays testing the effect of different concentrations of compounds on the $\mathrm{EC}_{50}$ value of CCK. Further experiments with these compounds revealed that compound 12 exhibited the most notable positive cooperativity with CCK. This cooperativity was maintained in cells with both high and low levels of receptor expression, with the amount of intrinsic agonist activity reduced in the setting of low receptor expression, consistent with Monod-WymanChangeux mechanism model, as has been described for M1 muscarinic acetylcholine receptor PAMs (Canals et al., 2012).
Similar to SR146131, the degree of PAM activity of compound 12 was predicted to be greater when enhancing the action of the partial agonist, CCK-OPE. These data are illustrative of probe dependency in allosterism that follows the Monod-Wyman-Changeux model, and is consistent with some $\mathrm{M}_{1}$ muscarinic acetylcholine receptor PAMs (Canals et al., 2012). This enhancement of a weak response of a partial agonist has also been recorded for other receptors such as the $\mathrm{GABA}_{\mathrm{B}}$ receptor, where the PAM exhibited an increase in $\mathrm{E}_{\max }$ along with increase in potency of a partial agonist, as opposed to only a left shift in $\mathrm{EC}_{50}$ for a full agonist (Maksay et al., 2000). Greater enhancement, relative to the glucagonlike peptide-1 (GLP-1), of oxyntomodulin, or the GLP-1 metabolite GLP-1(9-36)NH2 that are weak or partial agonists, has also been observed with small-molecule PAMs at the GLP-1 receptor (Koole et al., 2010; Wootten et al., 2012). It is important to note that CCK-OPE is not a natural metabolite of CCK, and therefore, these data do not hold physiologic importance; however, it is an important tool to understand the compound pharmacology. Based on these observations, it might be interesting to explore whether a combination therapy with a partial agonist and this type of PAM would have therapeutic advantages over use of only the PAM itself.

It is important to note that in the case of SR146131 and the structurally related compounds tested in this study, the observed potency tracked with the affinity of the compounds, resulting in PAM agonists with varying degrees of activity. For these compounds, the PAM activity is dependent on their ability to stabilize an active conformation of the receptor that provides a lower energy barrier threshold for activation by the cobound orthosteric peptide; this is linked to their intrinsic 


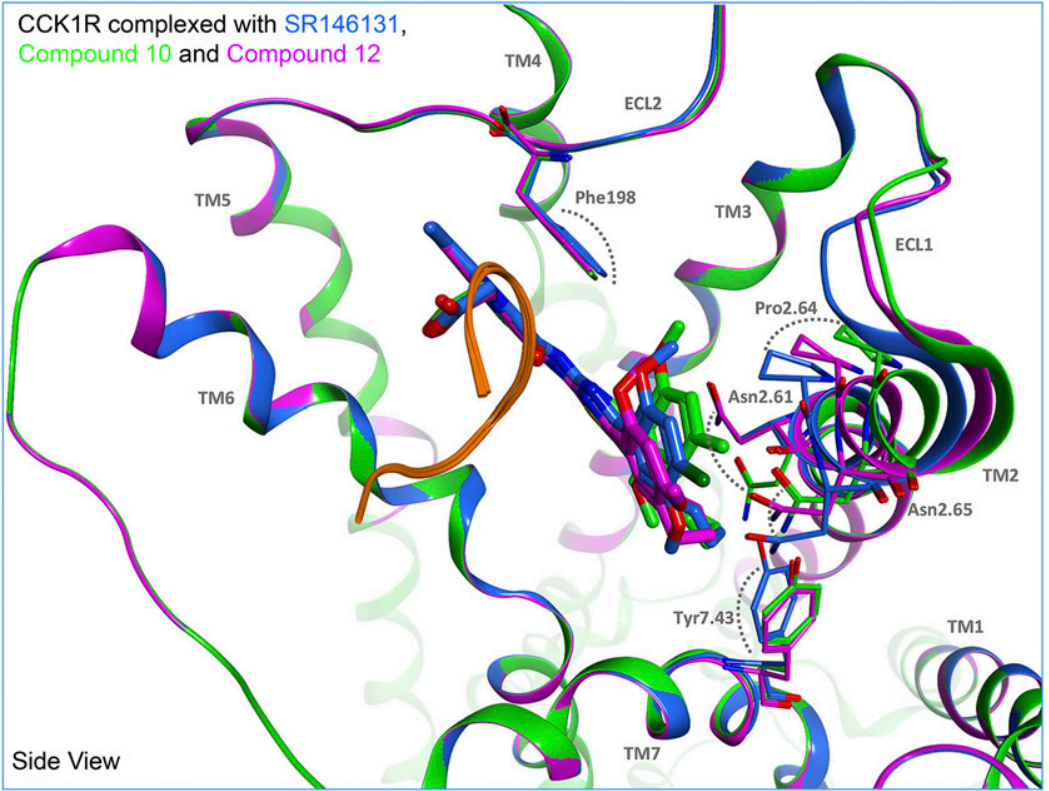

Fig. 12. Comparison between the Prime-mmGBSA calculations for SR146131 (ago-PAM), compound 10 (allosteric agonist), and compound 12 (PAM). CCK1R-SR146131 complex is shown in blue, CCK1R-compound 10 complex shown in green and CCK1R-compound 12 complex shown in magenta. Differences in side chain orientations in the vicinity of the substituted benzene ring are shown, whereas all other CCK1R residues are hidden for clarity. The CCK peptide is represented by an orange ribbon in all three cases. agonist activity. Prime-mmGBSA calculations on the complexes of small molecules bound to the receptor-peptide system show differences in $\Delta \mathrm{G}$ for these analogs, which is in qualitative agreement with their observed agonist or PAM agonist functional effects. However, as this series of compounds appear to follow the Monod-Wyman-Changeaux model of allosterism (Canals et al., 2012), it may be difficult to dissect the agonist activity from the PAM activity to achieve a pure PAM without intrinsic agonist activity. This phenomenon is also an issue for certain scaffolds of M1 muscarinic acetylcholine receptor PAM agonists (Miao et al., 2016), but can be overcome with alternative scaffolds (Khajehali et al., 2018).

Cinacalcet, a PAM of the calcium-sensing receptor, has rather small PAM activity for calcium at this receptor and had nonetheless been approved by the Food and Drug Administration in 2004. However, in this particular case, in which the serum concentrations of the endogenous ligand are tightly regulated, only a small change in the activity of the agonist is necessary, and even in some cases better than a large effect that could stimulate side effects. Of note, the small shift in potency by Cinacalcet is complemented by a higher cooperativity of the compound (Davey et al., 2012). However, such level of detail on fine-tuning the CCK1R function physiologically does not yet exist. Therefore, further experimentation is required to understand the desired magnitude of PAM activity at the CCK1R and will likely require identification of a novel scaffold with no intrinsic efficacy.

In conclusion, we report that SR146131 is a PAM agonist that binds to an allosteric pocket of the CCK1R (Harikumar et al., 2013). Based on the proposed binding pose for SR146131 within this pocket, we postulate that the methoxy groups are important for the agonist activity. By studying analogs of SR146131 with different phenyl substitutions both in vitro and in silico, we gained further insights into the mode of small-molecule ligand binding and receptor activation and the interactions between CCK1R, PAMs, and CCK that drive agonism and cooperative behavior. These insights are important for future drug design toward a PAM without intrinsic agonist activity that might be useful for the therapy of obesity.

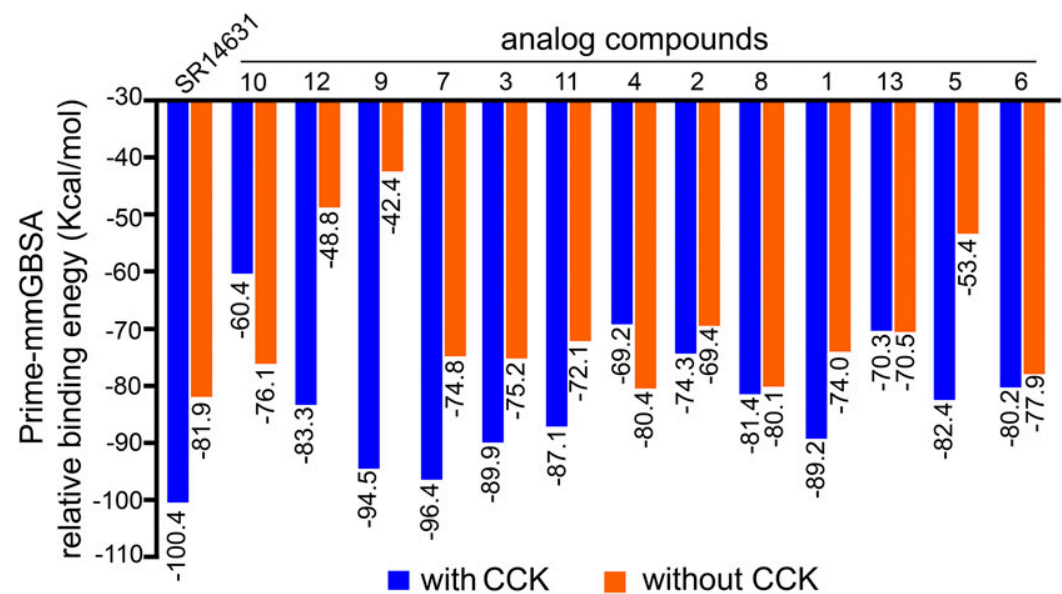

Fig. 13. Prime-mmGBSA values for all compounds in the presence or absence of CCK. Compounds in this graph are illustrated in the order of decreasing potency of agonist activity. Blue bars correspond to Prime-mmGBSA calculations in the presence of CCK peptide, whereas red bars correspond to the same calculations after removal of the CCK peptide. Prime-mmGBSA relative binding energies (in kilocalories per mole) are noted below each bar. 


\section{Acknowledgments}

We thank Sanofi for providing analogs of SR146131; Dr. David Thorpe for initiating this collaboration; and Dr. Maoqing Dong for help in manuscript preparation.

\section{Authorship Contributions}

Participated in research design: Desai, Mechin, Sexton, Christopoulos, Miller.

Conducted experiments: Desai, Mechin, Nagarajan, Lam, Nair.

Performed data analysis: Desai, Mechin, Nagarajan, Valant,

Wootten, Nair, Sexton, Christopoulos, Miller.

Wrote or contributed to the writing of the manuscript: Desai, Mechin, Nagarajan, Valant, Wootten, Lam, Orry, Abagyan, Nair, Sexton, Christopoulos, Miller.

\section{References}

Akgün E, Körner M, Gao F, Harikumar KG, Waser B, Reubi JC, Portoghese PS, and Miller LJ (2009) Synthesis and in vitro characterization of radioiodinatable benzodiazepines selective for type 1 and type 2 cholecystokinin receptors. $J \mathrm{Med}$ Chem 52:2138-2147.

Aquino CJ, Armour DR, Berman JM, Birkemo LS, Carr RA, Croom DK, Dezube M, Dougherty RW Jr, Ervin GN, Grizzle MK, et al. (1996) Discovery of 1,5-benzodiazepines with peripheral cholecystokinin (CCK-A) receptor agonist activity. 1. Optimization of the agonist "trigger." J Med Chem 39:562-569.

Ballesteros JA and Weinstein H (1992) Analysis and refinement of criteria for predicting the structure and relative orientations of transmembranal helical domains. Biophys $J$ 62:107-109.

Barker JL, Harrison NL, and Mariani AP (1986) Benzodiazepine pharmacology of cultured mammalian CNS neurons. Life Sci 39:1959-1968.

Berger R, Zhu C, Hansen AR, Harper B, Chen Z, Holt TG, Hubert J, Lee SJ, Pan J, Qian S, et al. (2008) 2-Substituted piperazine-derived imidazole carboxamides as potent and selective CCK1R agonists for the treatment of obesity. Bioorg Med Chem Lett 18:4833-4837.

Bignon E, Alonso R, Arnone M, Boigegrain R, Brodin R, Gueudet C, Héaulme M, Keane P, Landi M, Molimard JC, et al. (1999) SR146131: a new potent, orally active, and selective nonpeptide cholecystokinin subtype 1 receptor agonist. II In vivo pharmacological characterization. J Pharmacol Exp Ther 289:752-761.

Bowers KJ, Chow E, Xu H, Dror RO, Eastwood MP, Gregersen BA, Klepeis JL Kolossváry I, Moraes MA, Sacerdoti FD, et al. (2006) Scalable algorithms for molecular dynamics simulations on commodity clusters, in Proceedings of the ACM/ IEEE Conference on Supercomputing (SC06); 2006 Nov 11-17; Tampa, FL. IEEE Computer Society Press, Washington, DC.

Canals M, Lane JR, Wen A, Scammells PJ, Sexton PM, and Christopoulos A (2012) A Monod-Wyman-Changeux mechanism can explain G protein-coupled receptor (GPCR) allosteric modulation. J Biol Chem 287:650-659.

Cawston EE, Lam PC, Harikumar KG, Dong M, Ball AM, Augustine ML, Akgün E, Portoghese PS, Orry A, Abagyan R, et al. (2012) Molecular basis for binding and subtype selectivity of 1,4 -benzodiazepine antagonist ligands of the cholecystokinin receptor. J Biol Chem 287:18618-18635.

Chaudhri OB, Salem V, Murphy KG, and Bloom SR (2008) Gastrointestinal satiety signals. Annu Rev Physiol 70:239-255.

Cheng Y and Prusoff WH (1973) Relationship between the inhibition constant (K1) and the concentration of inhibitor which causes 50 per cent inhibition (I50) of an enzymatic reaction. Biochem Pharmacol 22:3099-3108.

Christopoulos A and Kenakin T (2002) G protein-coupled receptor allosterism and complexing. Pharmacol Rev 54:323-374.

Davey AE, Leach K, Valant C, Conigrave AD, Sexton PM, and Christopoulos A (2012) Positive and negative allosteric modulators promote biased signaling at the calcium-sensing receptor. Endocrinology 153:1232-1241.

Desai AJ, Dong M, Harikumar KG, and Miller LJ (2015a) Impact of ursodeoxycholic acid on a CCK1R cholesterol-binding site may contribute to its positive effects in digestive function. Am J Physiol Gastrointest Liver Physiol 309:G377-G386.

Desai AJ, Dong M, Harikumar KG, and Miller LJ (2016a) Cholecystokinin-induced satiety, a key gut servomechanism that is affected by the membrane microenvironment of this receptor. Int J Obes Suppl 6 (Suppl 1):S22-S27.

Desai AJ, Dong M, Langlais BT, Dueck AC, and Miller LJ (2017) Cholecystokinin responsiveness varies across the population dependent on metabolic phenotype Am J Clin Nutr 106:447-456.

Desai AJ, Dong M, and Miller LJ (2016b) Beneficial effects of $\beta$-sitosterol on type 1 cholecystokinin receptor dysfunction induced by elevated membrane cholesterol. Clin Nutr 35:1374-1379.

Desai AJ, Harikumar KG, and Miller LJ (2014) A type 1 cholecystokinin receptor mutant that mimics the dysfunction observed for wild type receptor in a high cholesterol environment. $J$ Biol Chem 289:18314-18326.

Desai AJ, Henke BR, and Miller LJ (2015b) Elimination of a cholecystokinin receptor agonist 'trigger' in an effort to develop positive allosteric modulators without intrinsic agonist activity. Bioorg Med Chem Lett 25:1849-1855.

Desai AJ, Lam PC, Orry A, Abagyan R, Christopoulos A, Sexton PM, and Miller LJ (2015c) Molecular mechanism of action of triazolobenzodiazepinone agonists of the type 1 cholecystokinin receptor: possible cooperativity across the receptor homodimeric complex. J Med Chem 58:9562-9577.

Ding XQ, Dolu V, Hadac EM, Holicky EL, Pinon DI, Lybrand TP, and Miller LJ (2001) Refinement of the structure of the ligand-occupied cholecystokinin receptor using a photolabile amino-terminal probe. J Biol Chem 276:4236-4244.
Ding XQ, Pinon DI, Furse KE, Lybrand TP, and Miller LJ (2002) Refinement of the conformation of a critical region of charge-charge interaction between cholecystokinin and its receptor. Mol Pharmacol 61:1041-1052.

Dong M, Lam PC, Pinon DI, Abagyan R, and Miller LJ (2009) Elucidation of the molecular basis of cholecystokinin peptide docking to its receptor using site-specific intrinsic photoaffinity labeling and molecular modeling. Biochemistry 48: 5303-5312.

Ehlert FJ (1988) Estimation of the affinities of allosteric ligands using radioligand binding and pharmacological null methods. Mol Pharmacol 33:187-194.

Elliott RL, Cameron KO, Chin JE, Bartlett JA, Beretta EE, Chen Y, Jardine PdaS, Dubins JS, Gillaspy ML, Hargrove DM, et al. (2010) Discovery of N-benzyl-2-[(4S) 4-(1H-indol-3-ylmethyl)-5-oxo-1-phenyl-4,5-dihydro-6H-[1,2,4] triazolo[4,3-a] [1,5]benzodiazepin-6-yl]-N-isopropylacetamide, an orally active, gut-selective CCK1 receptor agonist for the potential treatment of obesity. Bioorg Med Chem Lett 20:6797-6801.

Escrieut C, Gigoux V, Archer E, Verrier S, Maigret B, Behrendt R, Moroder L, Bignon E, Silvente-Poirot S, Pradayrol L, et al. (2002) The biologically crucial C terminus of cholecystokinin and the non-peptide agonist SR-146,131 share a common binding site in the human CCK1 receptor: evidence for a crucial role of Met-121 in the activation process. J Biol Chem 277:7546-7555.

Friesner RA, Banks JL, Murphy RB, Halgren TA, Klicic JJ, Mainz DT, Repasky MP, Knoll EH, Shelley M, Perry JK, et al. (2004) Glide: a new approach for rapid, accurate docking and scoring. 1. Method and assessment of docking accuracy. $J$ Med Chem 47:1739-1749.

Gaisano HY, Klueppelberg UG, Pinon DI, Pfenning MA, Powers SP, and Miller LJ (1989) Novel tool for the study of cholecystokinin-stimulated pancreatic enzyme secretion. J Clin Invest 83:321-325.

Gibbs J, Young RC, and Smith GP (1973) Cholecystokinin decreases food intake in rats. J Comp Physiol Psychol 84:488-495.

Gigoux V, Escrieut C, Silvente-Poirot S, Maigret B, Gouilleux L, Fehrentz JA, Gully D, Moroder L, Vaysse N, and Fourmy D (1998) Met-195 of the cholecystokinin-A receptor interacts with the sulfated tyrosine of cholecystokinin and is crucial for receptor transition to high affinity state. J Biol Chem 273:14380-14386.

Gigoux V, Maigret B, Escrieut C, Silvente-Poirot S, Bouisson M, Fehrentz JA, Moroder L, Gully D, Martinez J, Vaysse N, et al. (1999) Arginine 197 of the cholecystokinin-A receptor binding site interacts with the sulfate of the peptide agonist cholecystokinin. Protein Sci 8:2347-2354.

Giragossian C and Mierke DF (2001) Intermolecular interactions between cholecystokinin- 8 and the third extracellular loop of the cholecystokinin A receptor. Biochemistry 40:3804-3809.

Gouldson P, Legoux P, Carillon C, Delpech B, Le Fur G, Ferrara P, and Shire D (2000) The agonist SR 146131 and the antagonist SR 27897 occupy different sites on the human CCK(1) receptor. Eur J Pharmacol 400:185-194.

Hadac EM, Dawson ES, Darrow JW, Sugg EE, Lybrand TP, and Miller LJ (2006) Novel benzodiazepine photoaffinity probe stereoselectively labels a site deep within the membrane-spanning domain of the cholecystokinin receptor. $J \mathrm{Med}$ Chem 49:850-863.

Hadac EM, Ghanekar DV, Holicky EL, Pinon DI, Dougherty RW, and Miller LJ (1996) Relationship between native and recombinant cholecystokinin receptors: role of differential glycosylation. Pancreas 13:130-139.

Hadac EM, Pinon DI, Ji Z, Holicky EL, Henne RM, Lybrand TP, and Miller LJ (1998) Direct identification of a second distinct site of contact between cholecystokinin and its receptor. J Biol Chem 273:12988-12993.

Harikumar KG, Cawston EE, Lam PC, Patil A, Orry A, Henke BR, Abagyan R, Christopoulos A, Sexton PM, and Miller LJ (2013) Molecular basis for benzodiazepine agonist action at the type 1 cholecystokinin receptor. J Biol Chem 288: 21082-21095.

Hoshi H and Logsdon CD (1993) Both low- and high-affinity CCK receptor states mediate trophic effects on rat pancreatic acinar cells. Am $J$ Physiol 265: G1177-G1181.

Jacobson MP, Pincus DL, Rapp CS, Day TJ, Honig B, Shaw DE, and Friesner RA (2004) A hierarchical approach to all-atom protein loop prediction. Proteins 55: 351-367.

Ji Z, Hadac EM, Henne RM, Patel SA, Lybrand TP, and Miller LJ (1997) Direct identification of a distinct site of interaction between the carboxyl-terminal residue of cholecystokinin and the type A cholecystokinin receptor using photoaffinity labeling. J Biol Chem 272:24393-24401.

Jordan J, Greenway FL, Leiter LA, Li Z, Jacobson P, Murphy K, Hill J, Kler L, and Aftring RP (2008) Stimulation of cholecystokinin-A receptors with GI181771X does not cause weight loss in overweight or obese patients. Clin Pharmacol Ther 83:281-287.

Kennedy K, Gigoux V, Escrieut C, Maigret B, Martinez J, Moroder L, Fréhel D, Gully D, Vaysse N, and Fourmy D (1997) Identification of two amino acids of the human cholecystokinin-A receptor that interact with the N-terminal moiety of cholecystokinin. $J$ Biol Chem 272:2920-2926.

Khajehali E, Valant C, Jörg M, Tobin AB, Conn PJ, Lindsley CW, Sexton PM, Scammells PJ, and Christopoulos A (2018) Probing the binding site of novel selective positive allosteric modulators at the $\mathrm{M}_{1}$ muscarinic acetylcholine receptor. Biochem Pharmacol 154:243-254.

Kissileff HR, Pi-Sunyer FX, Thornton J, and Smith GP (1981) C-terminal octapeptide of cholecystokinin decreases food intake in man. Am J Clin Nutr 34:154-160.

Koole C, Wootten D, Simms J, Valant C, Sridhar R, Woodman OL, Miller LJ, Summers RJ, Christopoulos A, and Sexton PM (2010) Allosteric ligands of the glucagon-like peptide 1 receptor (GLP-1R) differentially modulate endogenous and exogenous peptide responses in a pathway-selective manner: implications for drug screening. Mol Pharmacol 78:456-465.

Labute P (2010) LowModeMD--implicit low-mode velocity filtering applied to conformational search of macrocycles and protein loops. J Chem Inf Model 50:792-800.

Latorraca NR, Venkatakrishnan AJ, and Dror RO (2017) GPCR dynamics: structures in motion. Chem Rev 117:139-155. 
Leach K, Sexton PM, and Christopoulos A (2007) Allosteric GPCR modulators: taking advantage of permissive receptor pharmacology. Trends Pharmacol Sci 28:382-389.

Li Y and Owyang C (1994) Endogenous cholecystokinin stimulates pancreatic enzyme secretion via vagal afferent pathway in rats. Gastroenterology 107:525-531.

Maksay G, Thompson SA, and Wafford KA (2000) Allosteric modulators affect the efficacy of partial agonists for recombinant GABA(A) receptors. $\mathrm{Br} J$ Pharmaco 129:1794-1800.

May LT, Avlani VA, Langmead CJ, Herdon HJ, Wood MD, Sexton PM, and Christopoulos A (2007) Structure-function studies of allosteric agonism at M2 muscarinic acetylcholine receptors. Mol Pharmacol 72:463-476.

Miao Y, Goldfeld DA, Moo EV, Sexton PM, Christopoulos A, McCammon JA, and Valant C (2016) Accelerated structure-based design of chemically diverse allosteric modulators of a muscarinic G protein-coupled receptor. Proc Natl Acad Sci USA 113:E5675-E5684.

Miller LJ and Desai AJ (2016) Metabolic actions of the type 1 cholecystokinin receptor: its potential as a therapeutic target. Trends Endocrinol Metab 27:609-619.

Nemeth EF, Heaton WH, Miller M, Fox J, Balandrin MF, Van Wagenen BC, Colloton M, Karbon W, Scherrer J, Shatzen E, et al. (2004) Pharmacodynamics of the type II calcimimetic compound cinacalcet HCl. J Pharmacol Exp Ther 308:627-635.

Pellegrini M and Mierke DF (1999) Molecular complex of cholecystokinin-8 and $\mathrm{N}$-terminus of the cholecystokinin A receptor by NMR spectroscopy. Biochemistry 38:14775-14783.

Powers SP, Pinon DI, and Miller LJ (1988) Use of N,O-bis-Fmoc-D-Tyr-ONSu for introduction of an oxidative iodination site into cholecystokinin family peptides. Int $J$ Pept Protein Res 31:429-434.

Rasmussen SG, DeVree BT, Zou Y, Kruse AC, Chung KY, Kobilka TS, Thian FS, Chae PS, Pardon E, Calinski D, et al. (2011) Crystal structure of the $\beta 2$ adrenergic receptor-Gs protein complex. Nature 477:549-555.
Sherman W, Day T, Jacobson MP, Friesner RA, and Farid R (2006) Novel procedure for modeling ligand/receptor induced fit effects. J Med Chem 49:534-553.

Smith GP, Jerome C, Cushin BJ, Eterno R, and Simansky KJ (1981) Abdominal vagotomy blocks the satiety effect of cholecystokinin in the rat. Science $\mathbf{2 1 3}$ : 1036-1037.

Smith GP, Jerome C, and Norgren R (1985) Afferent axons in abdominal vagus mediate satiety effect of cholecystokinin in rats. Am J Physiol 249:R638-R641.

Smith JP and Solomon TE (2014) Cholecystokinin and pancreatic cancer: the chicken or the egg? Am J Physiol Gastrointest Liver Physiol 306:G91-G101.

Totrov M (2008) Atomic property fields: generalized 3D pharmacophoric potential for automated ligand superposition, pharmacophore elucidation and 3D QSAR. Chem Biol Drug Des 71:15-27.

Totrov M and Abagyan R (1997) Flexible protein-ligand docking by global energy optimization in internal coordinates. Proteins 29 (Suppl 1):215-220.

Watts KS, Dalal P, Tebben AJ, Cheney DL, and Shelley JC (2014) Macrocycle conformational sampling with MacroModel. J Chem Inf Model 54:2680-2696.

Wootten D, Savage EE, Valant C, May LT, Sloop KW, Ficorilli J, Showalter AD, Willard FS, Christopoulos A, and Sexton PM (2012) Allosteric modulation of endogenous metabolites as an avenue for drug discovery. Mol Pharmacol 82:281-290.

Yin J, Babaoglu K, Brautigam CA, Clark L, Shao Z, Scheuermann TH, Harrell CM, Gotter AL, Roecker AJ, Winrow CJ, et al. (2016) Structure and ligand-binding mechanism of the human OX1 and OX2 orexin receptors. Nat Struct Mol Biol 23: $293-299$.

Address correspondence to: Dr. Laurence J. Miller, Mayo Clinic, 13400 East Shea Boulevard, Scottsdale, AZ 85259. E-mail: miller@mayo.edu 